\title{
33. STRUCTURE AND DYNAMICS OF THE GALACTIC SYSTEM (STRUCTURE ET DYNAMIQUE DU SYSTEMME GALACTIQUE)
}

\author{
PRESIDENT: L. Blitz (USA) \\ VICE-PRESIDENT: J.J. Binney (U.K.) \\ ORGANISING COMMITTEE: J.B.G.M. Bloemen (Netherlands), \\ C. Cesarsky (France), A. Fridman (Russia), \\ G. Gilmore (U.K.), A. Kalnajs (Australia), \\ F. Matteucci (Italy), M. Mayor (Switzerland), \\ M. Morris (USA), Y. Yoshii (Japan)
}

\section{Introduction}

This report covers work published approximately from July 1, 1991 to June 30, 1993. The main reference has been the Astronomy and Astrophysics abstracts; some of the sections were written using the last 6 volumes available to each writer. Consequently, some of the references date back to 1990 .

The intention has been to cover the field of galactic structure, and stellar dynamics. (Magneto)hydrodynamics as applied to the Milky Way system has also been included. There is some inevitable overlap not only with the work of other commissions, but within some of the sections of this report. However, work which is clearly in the purview of other commissions, notably Interstellar Medium (Commission 34) and Galaxies (Commission 28) has not been included. It has not been possible to provide an exhaustive summary of the work done on the subject of galactic structure in the past triennium, and the report provides a subjective view by the individual authors of the most important work in each subfield. Nevertheless, the past triennium has seen a great deal of exciting new work done in the field including the apparent discovery of compact objects by means of gravitational lensing within the Milky Way (MACHOs), as this volume went to press.

Thanks go to all of the authors who provided input on what turned out to be a very tight schedule, especially to Art Upgren, Jim Stone and John Howe who provided sections or parts of sections even though they are not on the organizing committee and to Jim Morgan of the University of Maryland who helped with the formatting of the camera ready manuscript.

\section{Basic Data}

\author{
Arthur R. Upgren \\ Wesleyan University
}

\subsection{Parallaxes}

\subsubsection{Trigonometric parallaxes from space}

The ESA Astrometric Satellite Hipparcos finally succumbed to the ravages of twice-daily passages through radiation belts, and its data collection ended in March 1993, almost four years after launch. Preliminary reports of its performance were made at the Galactic and Solar System Optical Astrometry Observation and Application Workshop held at Cambridge, U.K. in June 1993, by L. Lindegren and J. Kovalevsky. They report a median standard error in proper motion of 4.6 milliarcseconds (mas) per year and in parallax of 2.2 mas from the first half of the observations. 
They estimate errors from all observations will be about 2.2 mas/year and 1.8 mas for the proper motions and the parallaxes, the later representative of the true external errors. Thus the precision of its data equals or exceeds preliminary expectations. We are assured that the results will have a major impact on stellar and galactic astronomy for years to come when they are released in final form about 1996/97. In addition, about a million stars brighter than $B=11$ have been observed photometrically by the Tycho mission. A link of this system to the extragalactic reference frame is planned.

\subsubsection{Ground-based and other parallax observations}

The ongoing programs of the U.S, Naval, Allegheny, Van Vleck, Sproul and McCormick Observatories are continuing, with improvements in precision at several places. A. R. Upgren reports that the emphasis of the Van Vleck parallax program concentrates more fully on nearby metal-poor subdwarfs and stars reported to be of intermediate metallicity, that may represent a thick disk population. C.C. Dahn reports that the Naval program has completed parallaxes of about one thousand stars. Its mission has been extended to include bright $(\mathrm{V}<10)$ stars by using neutraldensity magnitude-compensating filters, and very faint stars in the $16<\mathrm{V}<22$ range by using a CCD detector. D. J. MacConnell of STI/CSC and W.H. Osborn of Central Michigan U. report first observations with the HST-FGS intended to determine parallaxes for high-velocity stars. R.B. Hanson (Lick Observatory) T. E. Lutz (Washington State U.) and W.F. van Altena (Yale) have nearly finished their joint project to improve the luminosity calibration of field subdwarfs by color and metal abundance, using data from the new edition of the Yale Parallax Catalogue.

\subsubsection{Catalogues of parallaxes and nearby stars}

The new fourth edition of the Yale Parallax Catalogue is being made ready for publication by van Altena, J.T. Lee and E.D. Hoffleit. It is scheduled to be sent to the L. Davis Press before the end of 1993. It contains 15,430 parallax observations for 7,888 stars. These numbers represent an increase over those given in the previous report in this series and elsewhere. Corrections between the various observatories, applied in earlier editions, have not been recommended by a number of investigators, especially since their physical cause cannot be identified, and hence will not be applied in this edition.

The third edition of the Catalogue of Nearby Stars (CNS3) is in final preparation by W. Gliese and H. Jahreiss. Its total of about 3800 stars represents a very large increase over the second edition of 1969 and its supplement of 1979. Part of the increase is due to an extension of the distance limit to 25 parsecs. As might be expected, most of the newly added stars are faint dwarf M stars.

Gliese, Jahreiss, Upgren and E.W. Weis are preparing a publication of the stars in the five lists of dwarf $\mathrm{K}$ and $\mathrm{M}$ stars found spectrophotometrically by A.N. Vyssotsky. Precise positions and proper motions as well as broad band photometry are now available for all of his 895 stars and stellar systems. At the Cambridge workshop cited above, Weis and Upgren reported a high velocity bias present in the Vyssotsky stellar sample. This is disturbing because this sample is often taken as the best unbiased stellar group representative of the galactic disk. The cause of the bias is not yet clear but analysis is continuing.

The Hipparcos Input Catalogue was published and circulated in 1992. Its seven volumes list the 118,322 stars for which positions, parallaxes and proper motions are to be obtained by the satellite. Accurate B1950 and J2000 positions, cross references and much other data for each star make this a useful standard reference. 


\title{
2.2 Proper Motions
}

The PPM Star Catalogue covering the northern celestial hemisphere was published in two volumes in 1991 by S. Roser and U. Bastian of ARI, Heidelberg. It contains the positions and proper motions of 181,731 stars north of $\mathbf{- 2 . 5}$ degrees for the equinox and epoch J2000. Similar data for the stars in the southern hemisphere has been obtained and will be published soon. This work contains data for 197,179 stars south of -2.5 degrees.

At Hamburg and the RGO the conventional plate adjustment solution and photographic photometry was completed for the CPC2; a catalogue containing 276,131 stellar positions and visual magnitudes for an average epoch of 1968 and an average standard error of 60 mas. The Pulkovo Observatory, in collaboration with the Sternberg Institute, ARI and Hamburg, the compilation of the proper motions of the FOCAT-S catalog was finished using AC and $\mathrm{CPC} 2$ data.

\subsection{Photometry and Stellar Classification}

Only one meeting covered the whole field of spectral classification during this triennium. This was "The MK Process at 50 Years: A Powerful Tool for Astrophysical Insight" sponsored by the Vatican Observatory and held at Tucson in September 1993 under the chairmanship of C. Corbally.

A very large number of studies were made of a very wide variety of stars and stellar types. They are reviewed in the report of Commission 45, among others. Those of the most general nature include a new spectral classification system by Keenan for the carbon stars bringing them into the revised MK system. Four subgroups of carbon stars are noted and designated R, N, CH and $\mathrm{Hd}$ (hydrogen deficient) $\mathrm{C}$ stars. New criteria have been identified that permit more realistic temperature sequences, as well as a luminosity classification for the first time.

Abt is classifying the 750 stars lacking MK classifications in the Bright Star Catalogue and a thousand members of visual multiple stars brighter than $\mathrm{B}=8$. Garrison is reviewing the MK standards over the entire HR Diagram. He is also classifying the nearest $800 \mathrm{G}$ dwarfs and the 1500 brightest stars in the sky. With the promise from Hipparcos of good parallaxes for many of these stars for the first time, the classifications of Abt and Garrison will be especially valuable as luminosity determinations.

A number of objective-prism surveys have been followed up by classification at higher dispersion. This was due in part to the increased sensitivity of current spectrographs and detectors. Much of this work is summarized in Contribution of the Van Vleck Observatory no. 12: Objectiveprism and Other Surveys.

New progress on catalogues includes the release by P. Renson, M. Gerbaldi and F.A. Catalano of their General Catalogue of Ap and Am stars, and a new catalogue by $\mathrm{G}$. Cayrel of $[\mathrm{Fe} / \mathrm{H}]$ determinations. The Institut d'Astronomie de l'Universite de Lausanne and the Observatoire de Geneve continue to maintain the General Catalogue of Photometric Data, with the Catalogue of Observations in the UBV System being kept up-to-date by Mermilliod. A new edition of the Catalogue of Am stars with known Spectral Types has been completed by Hauck.

\section{The Stellar Component}

\author{
Gerard Gilmore \\ University of Cambridge
}

The striking characteristics of studies of the stellar distribution in the Galaxy over the past triennium is the extension of statistical studies to include a very wide wavelength range, and to 
include kinematics. Fortunately, this burgeoning of activity is also matched by a burgeoning of excellent review articles and tutorial schools, as well as many sessions at regional IAU meetings. Thus extensive reviews cover the activity from many points of view, and will not be duplicated here.

One of the most significant publications has been the last planned paper in the Basle Halo Program "Model Comparison Program" (Fenkart \& del Rio, 1991, AASup, 89, 217). This major and long lasting survey has served as a model of modern statistical studies of Galactic structure, and their interpretation in terms of large scale Galactic structural properties. Important successor programs include those underway in Besancon, which incorporates both star counts and proper motion data (Bienayme et al., 1992, AA, 253, 389 and refs therein), a similar program based in Paris, at the MAMA group (Soubiran $A A, \mathbf{x x x x}$ ), that in Japan, utilizing star counts in many passbands in several fields (Yamagata \& Yoshii, 1992, $A J, 103,117$ ), and the north pole survey in photometry and proper motions by Majewski in the USA (1992, ApJS, 78, 87). Very considerable progress in this field is expected soon with the recent digitizations of the various Schmidt Sky Surveys, and the completion of the Lick and Yale proper motion surveys.

Extension of these optical surveys to the near infra-red has been a dramatic feature of late, with more promised with the recent release of the COBE data. Near infrared balloon, satellite and ground based surveys have been presented and analyzed by several groups. Studies of particular note include those by Blitz \& Spergel (1991, ApJ, 379, 631), Kent et al. (1992, ApJS, 78, 403 and 1992, ApJ, 387, 181), Ruelas-Mayorga \& Teague (1992, AASup, 93, 61 and 1992, AA, 259 118), Nakada et al. (1991, Nature, 353, 140), Terndrup et al. (1991, ApJ, 378, 742), and Weinberg $(1992, A p J L, \mathbf{3 9 2}, \mathrm{L67})$. A feature of most of these discussions is their concentration of possible (in fact likely) non-axisymmetric structure in the stellar distribution in the central bulge. An ability to detect and focus on such deviations from the basic large scale structure is indicative of the growing sophistication of current Galactic studies. the basic features are now agreed, and a higher degree of sophistication in modeling is becoming justified. The current state of infrared surveys is well reviewed by Boulanger and Desert (The Infrared and Submillimeter Sky after COBE, eds. Signore \& Dupraz, Kluwer, 1992).

Surveys of specific classes of identifiable objects have proered of late. Perhaps the most impressive such studies, both in the scale of effort required and the detailed analyses resulting, are the surveys for AGB stars in the Bulge. OH/IR surveys have been reported by Lindqvist et al. (1992, AASup, 92, 43 and 1992, AA, 259, 118) and are allowing extensive studies of the dynamics of the central stellar distribution (Weinberg et al., 1992, ApJ, 384, 81 provides one example).

Extension to other specific classes of tracer star, very often with specific dynamical aims in mind to complement the structural studies, has been achieved by many authors. Examples representing some of those studies include distant blue horizontal branch stars (Norris \& Hawkins, 1991, ApJ, 380, 104 and Arnold \& Gilmore, 1992, MNRAS, 257, 225), nearby AF stars (Knude, 1991, AA 249 88), X-ray stars (Kashyap et al., 1992, ApJ, 391, 667 and Favata et al., 1992, AA, 256, 86) and WR stars (Shara et al., 1991, AJ, 102, 716 and Alfaro et al., 1992, ApJL, 386, L47).

The picture of the Galaxy which is emerging from these many studies is detailed, justified, still uncertain, but testable against current and forthcoming reliable data. The many tutorial overview publications describing these results which have recently appeared include in particular two books in the ASP Conference Series, Galaxy Evolution: The Milky Way Perspective, ed. S. Majewski, and The Minnesota Lectures on the Structure and Dynamics of the Milky Way, ed. R. Humphreys. The proceedings of many more general conferences have appeared, with those of particular note including: The Globular Cluster - Galaxy Connection, eds. G. Smith and J. Brodie (ASP); Variable Stars and Galaxies, ed. B. Warner (ASP); Precision Photometry, eds. Davis Philip, Upgren \& Janes (L Davis Press); Eleventh European IAU Regional Meeting, eds. Sanchez \& Vazquez (CUP); The Stellar Populations of Galaxies eds. Barbuy \& Renzini (Kluwer). 
These conferences have been complemented by a major review in ARAA by Majewski (Galactic Structure Surveys and Evolution of the Milky Way, 1993, 31, 575). It is a sign of a healthy subject that the number of reviews papers included in these conferences is sufficient to provide an adequate number of references for a normal report. The volume of science included is such that only by reading those articles can justice be done to the subject.

\title{
3.1 Stellar Initial Mass and Luminosity Functions
}

In the past few years studies of the stellar mass and luminosity functions have been dominated by heroic surveys and analyses. For stars with mass above about one-half that of the Sun the field remains dominated by the authority of Scalo's 1986 review (Fund Cosmic Physics 11, 1). Very considerable progress has been made at lower masses however. New sky surveys have led to improved photometric studies (Tinney, Mould, \& Reid, 1993, ApJ, 396, 173) so that the apparent luminosity function for local disk stars is now apparently well defined to near the lowest luminous masses. All recent well calibrated surveys agree that the luminosity function declines at the lowest luminosities. It seems finally that long-standing suspicions that there is a substantial upturn in the space density of the lowest luminosity stars have been excluded from serious consideration. the apparent luminosity function is flat or declining at the lowest luminosities.

Conversion of the apparent luminosity function to a mass function requires detailed modeling of many parameters, especially the range in age, chemical abundance and binarism in the sample and in its calibrators. Kroupa et al. $(1993, M N R A S, \mathbf{2 6 2}, 545)$ quantify the effect of each variable. Kroupa et al., in agreement with all other analyses, deduce that the mass function for low mass stars is adequately described as a power law in number of stars per unit mass interval, with power law slope about 1.1; that is, very low mass stars are the most common by number. However, their contribution to the mass or the luminosity of a mass function integrated over a realistically wide range is negligible. The possibility that brown dwarfs dominate the local mass density seems conclusively discounted.

The most robust test of these models and conclusions comes from study of open clusters and young star forming regions. Major statistical studies by Bryja et al. (1992, ApJL, 388, L23), Simons \& Becklin (1992, ApJ, 390, 431), Hambly et al. (MNRAS, 253, 1), Stauffer et al. (AJ, 101, 980), among several other studies reach on average rather similar conclusions. An extension to the field stars of the Galactic halo (Richer \& Fahlman, 1992, Nature, 358, 383) while necessarily not extending to such low masses, further confirms the basic similarity of the stellar luminosity function in all environments.

While surprising and unexpected, as data and modeling improve, the similarity between determinations of the intrinsic stellar initial mass function in different environments tend to persist and surprise more than do the differences.

\section{The Gas and Dust Components}

\author{
Leo Blitz and John E. Howe \\ University of Maryland
}

\subsection{Surveys and Reviews}

Many surveys of the Galaxy have been reported during the past triennium, but the most notable change has been the large increase in infrared data from satellites (COBE (Wright, E.L., et al., 1991, ApJ, 381, 200], Spacelab, and the continuing analysis of IRAS data), and balloons (Okuda, H., Infrared Phys., 1991, 32, 365), as well as the traditional ground based radio surveys. There 
have also been a large number of meetings related to the distribution of gas and dust in the Milky Way. Publications from these meetings include The Milky Way as a Galaxy, R. Buser and I. King, eds.; The Interstellar Disk-Halo Connection in Galaxies, J.B.G.M. Bloemen, ed.; The Evolution of the Interstellar Medium, L. Blitz; ed.; and Back to the Galaxy, F. Verter and S. Holt, eds. Major new reviews of the $\mathrm{HI}$ and $\mathrm{CO}$ gas content of the Milky Way were written by Dickey and Lockman (1990, ARAA, 28, 215), and Combes (1991, ARAA, 29, 195).

Radio surveys include the Bell Labs HI survey (Stark, A.A., et al., 1992, ApJS, 79, 104) and the Pulkovo HI survey (Bystrova, N.V. \& Yagudin, L.I., Bull. Spec. Ap. Obs. 24, 107). Radio continuum surveys have been carried out at $6 \mathrm{~cm}$ at Green Bank (Langston, G.I., et al., 1990, ApJS, 72, 621), and Parkes (Griffith, M., et al., 1991, P.A.S.Aust., 9, 243), $11 \mathrm{~cm}$ at Effelsberg, (Reich, et al., 1990, AASup, 85, 633; Fürst et al., 1990, AASup, 85691,805 ), $20 \mathrm{~cm}$ at the VLA (Becker, R.H., et al., 1990, ApJ, 358, 485; Helfand, et al., 1992, ApJS, 80, 211), $21 \mathrm{~cm}$ at Effelsberg (Reich, et al., 1990, AASup, 83, 539), and $800 \mathrm{~cm}(38 \mathrm{MHz})$ at Ooty (Rees, N., MNRAS, 1990, 244, 233). Radio synthesis surveys at $21 \mathrm{~cm}$ and $74 \mathrm{~cm}(408 \mathrm{MHz})$ have continued at Penticton (Joncas, G. \& Higgs, L.A., 1990, AASup, 82, 113; Normandeau, M., et al., 1992, AASup, 92,63). The first results of a new $\mathrm{H} \alpha$ survey of the galactic plane have also recently been published (Le Coarer, E., 1992, $A A$, 257, 389).

\subsection{Rotation Constants, Kinematics, and Global Structure}

A fundamentally new method for deriving the rotation curve of the outer Milky Way was published by Merrifield (1992, $A J, 103,1552)$. Using the azimuthal variation of the angular variation of the atomic hydrogen scale height beyond the Sun, he showed that the rotation curve remains flat to $2.5 \mathrm{R}_{0}$. Dutra and Maciel, (1990, Rev. Mex. Astron. Ap., 21, 264), redetermined the rotation curve using type I, IIa, and IIb planetary nebulae, and showed that the curve determined from the first two types agrees with that determined form other population I tracers. Nincovic derived a new formula for the planar velocity dispersions containing an asymmetric drift term (1992, Ap. $S p$. Sci., 187, 159), and showed that it is possible to determine the slope of the circular velocity independent of the Oort constants.

Blitz \& Spergel $(1991, A p J, 370,205)$ argued that the asymmetries in the atomic hydrogen distribution are best modeled as the result of gas on elliptical streamlines which is the dynamical result of an $m=2$ distortion to the Galactic spheroid. Kuijken (1991, in Warped Disks and Inclined Rings around Galaxies, p. 159) preferred an $m=1$ distortion to the potential as the origin of the asymmetry, and while there is general agreement that the disk is non-axisymmetric, there is continuing debate about its nature (Kuijken \& Tremaine 1991, in Dynamics of Disk Galaxies, p.71 and 1994, ApJ, in press).

There seems to be more general agreement on the existence of a stellar bar in the central region of the Milky Way from an analysis of the dust distribution at $2.2 \mu \mathrm{m}$ (Blitz \& Spergel 1991, ApJ, 379, 631; see also appropriate sections on stars and dynamics in this report). From Spacelab data, Kent et al. (1991, ApJ, 378, 131; 1992, ApJ, 387, 181) deduced the axisymmetric properties of the bulge, and disk, but argued that a non-axisymmetric bulge is necessary to explain the peak velocities of the $\mathrm{CO}$ and the $\mathrm{HI}$ distributions within a few hundred parsecs of the center. Similar conclusions were reached from modeling of Gerhard (1992, Rev. Mod. Astron., 5, 14), and CO data of Nakai (1992, PASJ, 44, L27).

\subsection{Local Galactic Structure}

The past triennium has seen an expansion of the work done on local structures such as the cirrus and high-latitude molecular clouds. Although most of this work is more properly in the domain 
of interstellar medium studies, some is of relevance to studies of local galactic structure. For example, Verschuur (1991, Ap. Sp. Sci. has found evidence of large scale morphological waves in long HI filaments suggesting that the motion of the gas is controlled by magnetic fields within flux tubes. With other coworkers, Verschuur has also found evidence that the HI filaments, and the associated IR and CO emission can be offset from one another by as much as a degree (1992, $A p J, 390,514)$; it is unclear what this implies, however.

Blitz (1991, Molecular Clouds, p.49) has argued that based on kinematic evidence, there is a new class of molecular cloud characterized by a high negative velocity at high galactic latitude; three such clouds are known to exist. Lilienthal and Wennmacher $(1990, A A, \mathbf{2 3 5}, \mathrm{L} 13)$ have found a new upper limit of $240 \mathrm{pc}$ to the distance of a high latitude HI filament toward SAO 30359. Jahoda et al. $(1990, A p J, 354,184)$ have identified and observed the Galactic line of sight with the smallest HI column density: $<6.6 \times 10^{19} \mathrm{~cm}^{-2}$. Gamma-rays have been shown to correlate well with the Lynds dark cloud catalogue at intermediate latitudes (Feitzinger and Stüwe, 1990, MNRAS, 242 395). A rarefied tunnel in the interstellar medium which appears to be an extension of the local bubble has been mapped in the direction of $\beta \mathrm{C} \mathrm{Ma}$ and has been found to extend $300 \mathrm{pc}$ in that direction (Welsh, 1991, ApJ, 373, 556).

\subsection{Vertical Structure of the Disk}

A new word, 'worm', entered the literature, referring to vertical structures in the disk as a result of multiple supernovae. Worms are related to 'chimneys' which deposit their hot gas in the hot Galactic Corona. A catalogue of worms was published by Koo et al. (1992, ApJ, 390, 108). Theoretical studies of chimneys and the associated 'blowout' into the halo were published by $\mathrm{Li}$ and Ikeuchi $(1990, A p J S, 73,401)$, and by Igumentschchev et al. (1990, AA, 234, 396). Dougherty and McKenzie (1990, Proc IAU Symp. 140, p. 185), argued that for determination of the gas density far from the plane, galactic wind models should replace hydrostatic models.

Lockman and Gehman examined the vertical structure of the Galactic HI layer and concluded that the vertical distribution of the gas is supported by turbulence and that there are several distributions of atomic hydrogen with different central densities and scale heights (1991, ApJ, 382, 182). Blitz, on the other hand (1991, in Proc. IAU Symp. 144, p.41), found scant evidence for a molecular cloud population with a scale height greater than about $70 \mathrm{pc}$. In theoretical work by Franco et al. $(1991, A p J, 366,443)$, it was argued that radiation pressure can levitate the gas disk to high latitudes driving a soft Galactic fountain. The $z$ distribution of HI in the longitude range $210<l<275$ was investigated by Yudeva (1991, Bull. Spec. Ap. Obs., 27, 109). Churchwell et al. found that the vertical distribution of ultracompact HII regions is similar to that of $\mathrm{O}$ stars in the Galaxy $((1990$, AASup, 83, 119).

Reynolds (1991, ApJL, 372, L17), has shown that diffuse ionized gas is clumped into regions with an electron density of $0.08 \mathrm{~cm}^{-3}$, and a volume filling fraction $>0.2$ of a $2 \mathrm{kpc}$ thick ionized layer. This layer has become widely known as the 'Reynolds layer.' UV absorption line measurements support a model where warm ionized gas is corotating with the disk to heights of $1.5 \mathrm{kpc}$, but where corotation breaks down at $z$ heights between 1.5 and $3.5 \mathrm{kpc}$ (Sembach, et al., 1991, $A p J, 372,81$ ). Savage et al. measured the scale height of Al III to be $1.0 \mathrm{kpc}$ compared to 0.67 $\mathrm{kpc}$ for the HI scale height to the same set of stars $(1990, A p J, \mathbf{3 6 1}, 107)$.

\subsection{Spiral Structure}

Several studies of global tracers of Galactic spiral structure were made during the past triennium. Zongwei et al. (1991, ApJ, 378, 93) performed Monte Carlo simulations of the distribution of SNRs and HII regions to determine possible correlations with spiral arms or disk populations 
and conclude that SNRs are not necessarily concentrated in spiral arms in the numbers one would expect if they are preferentially born there. De-lin (1990, Acta Astrophys. Sin. 10, 235) compared the radial and longitudinal distributions of $\mathrm{HI}$ and $\mathrm{CO}$ at subcentral points at $b=0$ and $10^{\circ}<l<80^{\circ}$ with current spiral arm models and found that strong $\mathrm{CO}$ emission exists in the interarm regions, there is no evidence for stronger $\mathrm{HI}$ and $\mathrm{CO}$ emission at the subcentral points, and the $\mathrm{CO}$ emission in general does not trace the spiral arms delineated by $21-\mathrm{cm} \mathrm{HI}$ emission or HII regions. Calculations of the flow of interstellar gas into the gravitational potential well of a spiral density wave at a $5 \mathrm{kpc}$ galactocentric distance show that at gas densities of $0.05-0.2$ $\mathrm{cm}^{-3}$, a phase transition causes inhomogeneities along the wave which eventually forms individual clouds (Mishurov 1991, Astronomicheskii Zhurnal 68, 65).

GMCs in the outer arm of the Galaxy with sizes and masses similar to the largest clouds in the inner Galaxy were found in a recent CO survey at $b=+1^{\circ}$ and $65^{\circ}<l<71^{\circ}$ (Digel, Bally, and Thaddeus 1990, ApJL, 357, L29). The clouds lie at $R=12 \mathrm{kpc}$ and imply a total molecular mass for the Galaxy at $R>11 \mathrm{kpc}$ of $1-7 \times 10^{8} M_{\odot}$. An age gradient of HII regions and open clusters across and along the Sagitarrius-Carina spiral arm was found by Avedisova (1989, Astrophysics 30,83 ), as well as a decreasing pitch angle in the HII region/open cluster distribution correlated with the age of the objects. Murphy and May $(1991, A A, 247,202)$ show from CO kinematic data and correlations with optical and radio tracers that recent massive star formation has occurred in massive GMCs 1-2 kpc away, located behind the Vela SNR and associated with the Vela spiral arm.

\subsection{High Velocity Clouds (HVCs), Coronal Gas, Galactic Fountains and Winds}

Several large-scale surveys of the high-velocity clouds were conducted during the past triennium, yielding important new results on the distribution and properties of these objects. Colgan, Salpeter, and Terzian (1990, ApJ, 351, 503) searched for high-velocity emission and absorption features towards 63 continuum sources, detecting emission in 18 and absorption in none. They conclude that there can not be a significant mass of high-velocity $\mathrm{HI}$ which is undetected due to very low spin temperatures in low-density regions, and that there does not exist a large population of low column density HVCs. Wakker analyzed the results of a Dwingeloo whole-sky survey $\left(\delta>-17^{\circ}\right)$ for HVCs $(1991, A A, 250,499$; Wakker and van Woerden 1991, $A A, 250,509)$ and identifies ten different population of HVCs distributed in 17 complexes, with a slope of -1.5 in the differential mass spectrum. He finds that the HVCs concentrate in the Galactic plane and are uniformly distributed in longitude, but are usually at large distances from the solar neighborhood. Higher resolution Westerbork observations of six fields show much substructure, but that $70 \%$ of the emission is extended over scales larger than a few arcminutes, suggesting an extended warm background component (Wakker and Schwartz 1991, AA, 250, 484; Wakker, 1991, AASup, 90, 495). Wakker summarizes much of the HVC work in IAU Symposium 144 (p. 27).

Based on high resolution observations of $\mathrm{Na}$ I and $\mathrm{Ca}$ II lines toward nine stars in the direction of the Chain $A$ high-velocity complex, Lilienthal and Meyerdierks (1990, $A A, \mathbf{2 4 0}, 487)$ set a lower limit of $200 \mathrm{pc}$ above the plane for the height of the complex. Meyer and Roth (1991, ApJL, 383, L41) detect weak Ca II absorption features in high resolution spectra towards SN 1991T in the galaxy NGC 4527 and attribute the features to low column density Galactic HVCs. HI emission spectra at $21 \mathrm{~cm}$ taken towards the Large Magellanic Cloud show high-velocity components well outside the LMC boundary and identify this gas as a local Galactic component (de Boer, Morras, and Bajaja 1990, $A A, \mathbf{2 3 3}, 523$ ).

A number of observational and theoretical studies of Galactic outflow and inflow phenomena were reported. Mirabel (1991, IAU Symposium 144, 89) and Mirabel and Morras (1990, ApJ, 
356,130 ) found evidence for an infalling stream of HVCs towards the Galactic anticenter which interacts with the ambient gas, producing shocks and an energetic "anticenter supershell" of HI. Globally, the Galaxy accretes $0.2-0.5 M_{\odot} /$ yr of extragalactic $\mathrm{HI}$ in addition to a general disk-halo gas circulation (Mirabel 1989, IAU Colloquium 120, p. 396). Meyerdierks proposes that the North Celestial Pole Loop is the result of the expansion of a cavity produced by an infalling $350 M_{\odot}$ halo cloud which has already collided with the Galactic disk (1991, $A A, 251,269)$. Meyerdierks also concludes on the basis of HI observations that an intermediate-velocity cloud in the Chain $A$ stream is the result of a high- and low-velocity cloud collision $(1992, A A, 253,515)$. Kahn (1989, IAU Colloquium 120, p. 474) proposes that Type II supernovae remnants generate the gas and energy driving a Galactic fountain of intercloud gas. The infalling gas is seen as intermediate velocity clouds. Houck and Bregman $(1990, A p J, 352,506)$ show that clouds can be formed from the fountain flow but the details of the formation depend on whether the gas cooling time is longer or shorter than the sound crossing time in the halo gas. Shapiro and Benjamin (1989, PASP, 103, 923) show that photoionization due to ionizing photons produced in the Galactic fountain flow itself is sufficient to reproduce the observed column densities of ionized metals in the flow. Spitzer and Fitzpatrick (1992, ApJL, 391, L41) observed C IV and Si IV absorption features towards an O star $1500 \mathrm{pc}$ above the plane and conclude that the velocities of the features show they are produced in infalling gas.

$\mathrm{Li}$ and Ikeuchi $(1992, A p J, 390,405)$ use a two-diminsional hydrodynamic code to model the Halo gas. Gas of temperature $0.5-1 \times 10^{6} \mathrm{~K}$ and density $10^{-3}-10^{-2} \mathrm{~cm}^{-3}$ reproduces many of the observed ionized metal column densities. Lazaryan and Chibisov (1991, Sov. Astron. Let., 17,208 ) propose a novel method for measuring the size of the Galactic radio halo based on a statistical analysis of synchrotron emission fluctuations. Cosmic rays may be important in driving a net outflow (1 $M_{\odot} \mathrm{yr}^{-1}$ in the present epoch) of hot interstellar gas from the Galactic disk (Breitschwerdt, McKenzie, and Völk 1991, AA, 245, 79).

\subsection{The Distribution and Properties of the Gas in the Plane}

A review of the structure of the Milky Way as seen in molecular emission is given by Thaddeus and Stacy (1990, The EGRET Science Symposium, p. 5). However, Adler and Roberts (1992, ApJ, 384,95 ) show cause for caution in identifying molecular cloud complexes from position-velocity diagrams and of assigning distances to them based on model rotation curves. Simulations of cloud complexes in model spiral galaxies show that many apparent correlations in position-velocity diagrams are in fact chance line-of-sight superpositions. Waller and Tacconi-Garman (1992, ApJS, $80,305)$ estimate distances to local CO clouds using the Massachusetts-Stony Brook CO survey data, and also find over 2100 lines of sight free of significant CO emission, useful as regions to study the diffuse intercloud medium and as windows for extragalactic observations. Characteristics of large-scale details in the Pulkovo HI sky survey are tabulated by Bystrova and Kozyrev (1990, Bull. Spec. Astrophys. Obs. 29, 106). Delin (1992, Acta Atrophys. Sin. 12,13) finds in a statistical study of the large-scale correlations between $\mathrm{CO}$ and $\mathrm{HI}$ between $11<l<45$ that the $\mathrm{CO}$ and $\mathrm{HI}$ emissivities are linearly uncorrelated, even in regions corresponding to spiral arms in some models. Yasutomi and Tatematsu $(1990, P A S J, 42,517)$ numerically modeled the dynamical evolution of GMCs in the disk and found that dynamical friction causes the GMCs to spiral in to the Galactic center. A minimum in the infall velocity occurs at $R=5 \mathrm{kpc}$, creating the $5 \mathrm{kpc}$ molecular ring on a time scale of about $10^{9} \mathrm{yr}$. Millimeter continuum observations from Antarctica of several southern clouds revealed an excess of emission at the longer wavelengths (Andreani et al., 1991, $A p J, 375,148)$, attributed to emission from a cold dust component coexisting with the warmer dust seen in the IRAS survey. In the first of what will undoubtedly be one of many studies of the large-scale Galactic distribution of ionized carbon ([C II]) emission, Shibai et al. (1991, ApJ, 374, 
522) show that the [C II] and $\mathrm{CO}$ distributions are well correlated and that the total luminosity of the [C II] emission in the inner Galactic disk amounts to about $0.4 \%$ of the far-IR luminosity. They conclude that the [C II] is largely emitted from dense, partially-ionized photodissociated skins of molecular clouds traced by the $\mathrm{CO}$, although extended low-density ionized gas may also contribute.

A wealth of information continues to be gleaned from the IRAS survey. Vedi and Walker (1991, MNRAS, 249, 729) derive values of $A_{V}=0.05$ towards the North Galactic Pole and $A_{V}=0.08$ towards the South Galactic Pole. Bloemen, Duel, and Thaddeus $(1990, A A, 233,437)$ decompose $60 \mu \mathrm{m}$ and $100 \mu \mathrm{m}$ maps, which trace the dust emission, into components associated with atomic (HI) and molecular (CO) gas. Stecker, Harding, and Skibo (1991, AIP Conf. Proc. No. 220, 20) find that the dominant feature in the radial distribution of IRAS far-IR emission coincides with the $5 \mathrm{kpc}$ molecular ring. Spiral arm features are also apparent, and a strong correlation exists between the far-IR emission and $\mathrm{CO}$ and $\gamma$-ray emission.

Diplas and Savage $(1991, A p J, \mathbf{3 7 7}, 126)$ studied the distribution of $\mathrm{HI}$ in the outer Galaxy from the Bell Labs HI survey data. They identify a confined component which exists only within $R \sim 18$ $\mathrm{kpc}$ and an extended component which exists out to $25-30 \mathrm{kpc}$ in some directions. The average $z$ distance of the $\mathrm{HI}$ in the direction of the Galactic warp is $\sim 4 \mathrm{kpc}$ at $R \sim 24 \mathrm{kpc}$. Sodroski (1991, $A p J, 366,95)$ analyzed outer Galaxy GMC properties from the Goddard-Columbia Galactic plane $\mathrm{CO}$ survey and finds size and mass distributions similar to inner Galaxy GMCs, but the $\mathrm{H}_{2}$ column density to $\mathrm{CO}$ intensity ratio $\left(6.0 \times 10^{20}\right.$ molecules $\left.\mathrm{cm}^{-2} \mathrm{~K}^{-1} \mathrm{~km}^{-1} \mathrm{~s}\right)$ is $2-3$ times greater than for inner Galaxy GMCs. Fich and Silkey $(1991, A p J, 366,107)$ derived high nitrogen abundances in $18 \mathrm{HII}$ regions at $R=12-18 \mathrm{kpc}$. Wouterloot et al. $(1990, A A, \mathbf{2 3 0}, 21)$ surveyed 1302 outer Galaxy IRAS point sources and found that 1077 have associated $\mathrm{CO}$ emission. Kinematic distances place these clouds out to $R=20 \mathrm{kpc}$ and indicate that star formation is presently occurring in the outer Galaxy. The cloud distribution also shows the warped-disk shape seen in HI.

The radial distribution of diffuse ionized gas was investigated by Frail et al. (1991, ApJ, 382, 168) using mean electron density measurements towards 18 pulsars. They find that $n_{e}$ in the inner Galaxy is about 3 times that found locally, and propose that the warm ionized medium is primarily heated by $\mathrm{O}$ stars. The distribution of plasma turbulence was found by Hajivassiliou (1992, Nature $\mathbf{3 5 5}, 355)$ to agree morphologically with the soft X-ray background, possibly suggesting that the solar neighborhood is enveloped in an ionized bubble from a nearby supernova explosion. A warp in the outer Galaxy at $270^{\circ}<l<300^{\circ}$ is also seen in ionized gas (Azcárate, Cersosimo, and Colomb 1990, Rev. Mex. Astron. Atrofis, 20, 75). Cersosimo (1990, IAU Colloq. No. 125, 237) reports evidence for diffuse ionized gas in the $3 \mathrm{kpc}$ arm, primarily but not exclusively associated with Pop I material, and some evidence of in interarm ionized gas connecting giant HII complexes in the Scutum-Crux and Norma arms. Lockman (1990, IAU Colloq. No. 125, 225) presents a Green Bank HII region survey, used with Southern Hemisphere data to investigate the radial distribution of HII regions. Oskanyan (1989, Astrophysics 30,75) shows that the distribution of Galactic HII regions shows a correlation with the distributions in Sc and SBc spirals, and estimates that the mass of neutral gas formed from "extinct" HII regions could be about $65 \%$ of the total mass of Galactic HI, if the lifetime of HII regions is only $6 \times 10^{5} \mathrm{yr}$.

\subsection{Galactic magnetic fields}

Heiles (1990, IAU Symp. No. 140,35) reviewed the status of magnetic field strength measurements in $\mathrm{HI}$ regions and finds that the magnetic field pressure dominates or is comparable to other pressures in some dark clouds and in some HI shells. Trimble (1990, IAU Symp. No. 140, 29) also reviewed the observational parameters of the Galactic magnetic field at the same meeting. Field directions derived from Zeeman effects do not correlate well with those derived from Faraday 
rotation measures. Rand and Kulkarni (1990, IAU Symp. No. 140,44) model the local magnetic field from available pulsar rotation measures and find a local field strength of $1.6 \pm 0.2 \mu \mathrm{G}$ toward $l=96^{\circ}$ and a field reversal $600 \mathrm{pc}$ toward the inner Galaxy. In the first Galactic quadrant, Clegg and Cordes (1990, IAU Symp. No. 140,47) find evidence for field reversals along several lines of sight, and for a structure at $l>60^{\circ}$ and a distance more than $7 \mathrm{kpc}$ causing excess rotation measures. This structure is also seen in Effelsberg $11 \mathrm{~cm}$ data (Junkes, Fürst, and Reich 1990, IAU Symp. No. 140,63), and confirmed in later measurements by Clegg et al. (1992, ApJ, 386, 143).

Andreasyan and Makarov (1989, Astrophysics 30, 101) give a detailed description of field strengths and directions in spiral arms in the solar neighborhood and out to distances of 5-7 kpc. Independent studies of optical stellar polarization (Andreasyan and Makarov 1989, Astrophysics 31, 560) and pulsar rotation measures (Costa, Hamilton, and McCulloch 1991, Proc. Astron. Soc. Aust. 9, 131) both conclude that the Galactic field is concentrated in and directed along the spiral arms. Cordes, Clegg, and Simonetti $(1990$, IAU Symp. No. 140,55) report that smallscale fluctuations in the Galactic magnetic field suggest that the energy in the turbulent magnetic component is comparable to the energy in the smoother large-scale field.

\title{
5 The Galactic Center
}

\author{
Mark Morris \\ University of California at Los Angeles
}

Improving techniques in radio, infrared, $\mathrm{X}$ - and gamma-ray astronomy are leading to an increasingly clear picture of the interplay of the various components of this region. Blitz et al. (1993 Nature, 361, 417) provided a recent review of many of the topical areas in Galactic center research.

\subsection{The Stellar Cluster}

During the past three years, progress has been made in defining the properties of the central stellar cluster of the Galaxy, particularly in the inner parsec. However, many of the most important questions remain unanswered. On scales of hundreds of parsecs, it appears that the most luminous stars are concentrated toward the nucleus such that they occupy a thick disk extending about 100 pc to either side of the Galactic plane (Catchpole et al., 1990, MNRAS, 247, 479; Lindquist et al., 1992, AA, 259, 118; Haller 1992, PhD Thesis, Univ. Arizona). Apparently, the Galactic center has repeated episodes of star formation in which massive (greater than a few $\mathrm{M}_{\odot}$ ) stars are formed. It is not clear how these presumably massive stars could have formed such a thick distribution during their lifetimes. Lindqvist et al. (1992) point out that OH/IR stars follow the integrated 2 $\mu \mathrm{m}$ light, and are therefore useful tracers of the kinematics of the central cluster. Using a sample of $134 \mathrm{OH} / \mathrm{IR}$ stars lying close to the Galactic center (Lindqvist et al., 1992, AASup, 92, 43), they observe the Galactic rotation, and deduce a velocity dispersion of about $75 \mathrm{~km} \mathrm{~s}^{-1}$. The mean values depend on the expansion velocity of the circumstellar envelopes of the $\mathrm{OH} / \mathrm{IR}$ stars, which, in turn, reflects the stellar masses. More massive stars, having higher outflow velocities, are more concentrated to the galactic plane, and have a smaller velocity dispersion. Angular broadening measurements of $20 \mathrm{OH} / \mathrm{IR}$ stars near the Galactic center were reported by van Langevelde et al. (1992, ApJ, 396, 686), and they used these measures to derive the distribution of interstellar scattering. A region of relatively large scattering was found to be nearly centered on Sgr A*, possibly as the result of density and turbulence enhancements at small galactocentric radii.

The question of of how star formation proceeds near the Galactic center was considered by Morris $(1993, A p J, 408,496)$. There are many factors inhibiting star formation there, or at least 
raising the Jeans mass, including strong tidal forces, strong magnetic fields, and large turbulent motions, so if stars do form within $100 \mathrm{pc}$ of the Galactic center (and certainly within a few parsecs), they may tend to be quite massive. Massive stars forming within about $4 \mathrm{pc}$ will leave remnants which can undergo gravitational settling and thereby sink to the inner 0.1-pc core in less than a Hubble time. As much as $10^{6} \mathrm{M}_{\odot}$ of black hole remnants could have been gathered into the core by this process. Whether these remnants merged to form a single object, or whether they exist as a tight cluster occasionally interacting with stars by mergers remains an open question. Moneti et al. (1992, MNRAS, 258 705) explored a number of IRAS sources near the Galactic center in the near-IR, and found a number of embedded sources which they regard as early-type ZAMS stars, so they conclude that star formation is ongoing on scales of 10 to $100 \mathrm{pc}$ around the Galactic center.

\subsection{Emission Line Stars and Winds}

A number of the luminous stars in the Galactic center were found by Krabbe et al. (1991, ApJL, 382 L19; see also Burton and Allen 1992, Proc. ASA 10, 55) to display near-infrared emission lines, most notably those of helium. It now appears that the collective winds from these emission line stars account for the "broad-line" region that had previously been observed to be more or less coincident with the central cluster of luminous blue objects comprising IRS16.

The winds from the IRS16 complex interact with the nearby red supergiant IRS7, creating a prominent cometary tail on this star directed away from the wind sources (Yusef-Zadeh and Morris 1991, ApJL, 371, L59; Serabyn et al., 1991, ApJ, 378, 557; Yusef-Zadeh and Melia 1992, $A p J L, \mathbf{3 8 5}, \mathrm{L} 41)$. The tail shows a clear gradient in its velocity field, which provides useful constraints on the star's orbit and the interaction of the wind with the star (Serabyn et al., 1991). The winds emerging from the IRS 16 complex also appear to be interacting with nearby plasma, perhaps leading to a waviness in the "northern arm" and to a compression of the plasma there and in the "bar" which may cause enhanced emission at mid-IR wavelengths (Yusef-Zadeh and Wardle 1993, ApJL, 405, 584). These authors also assess the earlier suggestion of Yusef-Zadeh et al. $\left(1990\right.$, Nature, 348, 45) that $\mathrm{Sgr} \mathrm{A}^{*}$, if it is a massive compact object, might be focusing the nuclear winds into the plasma "bar", forming the apparently evacuated "mini-cavity" (Wardle and Yusef-Zadeh 1992, Nature, 357, 308). The mini-cavity has also been found to be a strong source of iron-line emission by Eckart et al. (1992, Nature, 355, 526).

A model for the combined winds from a cluster of stars in the central $0.8 \mathrm{pc}$ was presented by Chevalier (1992, ApJL, 397 L39). He suggests that the wind passes through a shock at a radius of a few pc, and fills a hot bubble on a scale of $100 \mathrm{pc}$. The interaction of the collective winds with the ambient magnetic field might lead to some of the magnetized structures observed near the center.

\subsection{The Radio Arc and Other Polarized Radio Structures}

The Galactic center radio Arc remains an enigmatic structure, with less than complete consensus about its nature, other than that it is clearly a magnetic feature. Lesch and Reich (1992, AA, 264, 493) noted the slightly inverted radio spectrum of the linear filaments of the Arc, and proposed that the emission arises from monoenergetic particles which have been accelerated by magnetic reconnection at the interface between neighboring filaments. Sofue et al. $(1992, P A S J, 44,367)$ mapped the G0.18-0.04 region of the Arc interferometrically at $43 \mathrm{GHz}$, and concluded that the spectrum of the nonthermal radio filaments turns over between 4.7 and $43 \mathrm{GHz}$. The flat, thermal spectrum of G0.18-0.04 is confirmed.

Far-infrared polarization measurements of the thermal, arched filaments of the Arc were re- 
ported by Morris et al. (1992, ApJL, 399, L63). The degree of polarization is large, and the small dispersion of the directions of the polarization E-vectors implies that the field is uniform on scales of 1 to $10 \mathrm{pc}$. The inferred direction of the magnetic field in the arched filaments is parallel to the filaments, perhaps as a result of sheer within the cloud.

The Radio Arc extends over a full $2^{\circ}$ in Galactic latitude, according to Haynes et al. (1992, AA, 264, 500). These authors also describe $3.5-\mathrm{cm}$ observations of other polarized features extending perpendicular to the Galactic plane, including one which coincides with a previously reported radio "Thread" and another which coincides with the Western leg of the so-called "Galactic Center Lobe".

A new radio filament was reported by Gray et al. (1991, Nature, 353, 237). This one, located further from the center than any other at $l=359.1$, is unusual in having a few kinks, or cusps, along its length. As in the other "threads", the emission is polarized, and the structure appears to be magnetic in character.

\subsection{Interstellar Gas and Dust: Sgr A West and the Circumnuclear Disk}

The kinematics of gas in Sgr A West were investigated by several groups. Herbst et al. (1993, AJ, 105, 956) imaged Sgr A West in the $\mathrm{Br}-\gamma$ line with relatively high spatial and spectral resolution. They identify a continuous, coherent stream of gas starting with the northern arm, and extending around the IRS16/SgrA* complex into the so-called "bar". They model the kinematics of this feature in terms of orbital motion in the field of a central point mass. Depoy $(1992, A p J, 398$, 512) reported that the distribution of forbidden FeII emission at 1.644 and $1.533 \mu \mathrm{m}$ in $\mathrm{Sgr} A$ West is similar to that of NeII and $\operatorname{Br} \alpha$. The iron line emission, relative to $\operatorname{Br} \alpha$, is quite strong compared to normal HII regions.

Several studies have been carried out recently of a newly found, high-velocity ( $v_{L S R}=-170$ to $-190 \mathrm{~km} \mathrm{~s}^{-1}$ ) neutral cloud superimposed upon, and foreground to, Sgr A*. Marr et al. (1992, $A p J L, 400, \mathrm{~L} 29)$ observed $\mathrm{HCO}^{+}$absorption in this cloud with a millimeter interferometer, and concluded that some of this gas may be mixed in with the ionized gas of the "bar" in Sgr A West. Pauls et al. (1993, ApJL, 403, L13) observed formaldehyde absorption in this high-velocity cloud, and they place it within the central 1-pc cavity of the circumnuclear disk.

The 1 to $7 \mathrm{pc}$ circumnuclear disk (CND) was studied in increasing detail in the past triennium. Davidson et al. $(1992, A p J, 387,189)$ produced 50 and $100-\mu \mathrm{m}$ maps of the CND made with the Kuiper Airborne Observatory with high spatial resolution. They offer models for the heating of the CND from its interior, and estimate the central luminosity to be 1 or $2 \times 10^{7} L_{\odot}$. Also, they find that a substantial quantity of dust is emitting from within the central cavity of the CND. Jackson et al. $(1993, A p J, 402,173)$ carried out a variety of far-IR and radio spectroscopic observations of the inner 2 parsecs, and conclude that the CND is a nearly complete ring of clouds, with other kinematic components superimposed upon it along the line of sight. Inside the central cavity of the CND, Jackson et al. find evidence for several hundred $\mathrm{M}_{\odot}$ of dense neutral gas, presumably the same gas seen in the cavity by Davidson et al.; they find that the northern arm of Sgr A West is an ionized rim upon this neutral gas, produced by the ultraviolet radiation from the IRS16/Sgr $\mathrm{A}^{*}$ complex. The previously known molecular hydrogen emission at the inner edge of the CND was imaged by Burton and Allen (1992, Proc. ASA 10,55), who concluded that UV excitation is to be preferred over shock excitation as a means of accounting for the emission.

The heating and thermal equilibrium of the ionized gas in Sgr A West and the CND were considered by Maloney et al. (1992, ApJ, 401, 559). They deduce that $7 \times 10^{6} \mathrm{~L}_{\odot}$ is needed to account for the observed temperature and emission measure distribution in the "bar". This is comparable to the bolometric luminosity inferred from IR measurements, so the cooling emission 
from the hot gas near the center accounts for a major fraction of the bolometric luminosity emerging from this region.

\subsection{Interstellar Gas and Dust: 10 to 1000 pc}

It was pointed out by Spergel and Blitz $(1992$, Nature, $\mathbf{3 5 7}, 665)$ that the large, internal velocity dispersions in Galactic center clouds reflects a very high gas pressure, and that this gas pressure has a strong effect on the evolution of the medium. The hot, $x$-ray emitting gas found in the Galactic center is another indication of a high pressure. It should not be forgotten that magnetic pressures are at least comparable, and may in fact dominate the pressure balance in the Galactic center.

Some studies of individual molecular clouds in the Galactic center have appeared recently. A number of $\mathrm{OH}$ and $\mathrm{H}_{2} \mathrm{O}$ masers were observed in Sgr B1 by Mehringer et al. (1992, ApJL, 401, L87). They seem to indicate the ongoing formation of low- to intermediate-mass stars. An apparently expanding molecular cloud system was found to ring the nonthermal radio shell G359.1-0.5 (Uchida et al., 1992, ApJ, 398, 128). Uchida et al. present a model in which the expanding ring was initiated by the winds, radiation, and supernova ejecta from a massive central cluster of early-type stars. In a related paper, Uchida et al. $(1992, A J, 104,1533)$ describe a VLA study of $\mathrm{HI}$ absorption across G359.1-0.5 and some of the nearby radio sources. The HI absorption line velocities place G359.1-0.5 within the Galactic center region, along with the filamentary, radio "Snake", but a curious, quasi-axisymmetric nonthermal source referred to as the "Mouse" appears to be a foreground object.

The HII complexes Sgr D and Sgr E were studied in the radio continuum and recombination lines by Liszt $(1992, A p J S, \mathbf{8 2}, 495)$. He concluded that Sgr D is an HII region/SNR pair, while $\mathrm{Sgr} \mathrm{E}$ is a cluster of compact HII regions centered on recently-formed, massive stars.

The Galactic center annihilation line source, 1E1740.7-2942, aroused a great deal of attention from $\mathrm{X}$ - and $\gamma$-ray astronomers during the triennium. It is located about $100 \mathrm{pc}$ in projection away from the nucleus. Here, we note only a possible physical association between this potential stellarmass black hole and a molecular cloud of the Galactic center population. Bally and Leventhal (1991, Nature, 353, 234) and, with higher spatial resolution, Mirabel et al. (1991, AA, 251, L43) both reported that the high-energy source lies near the peak of a molecular cloud of intermediate density, and both groups considered that the source may be fueled by accretion of cloud material onto a single, compact object. In another study using the VLA, Mirabel et al. (1992, Nature, 358, $215)$ report the detection of radio jets emanating from a presumably compact and variable core in 1E1740.7-2942, allowing those authors to terms this object a "microquasar". Inasmuch as the jets may contain a pair plasma, the annihilation might be taking place in the radio lobes, where the positrons are deposited in the ambient medium.

A few large-scale surveys of molecular emission appeared in the recent triennium. Burton and Liszt $(1992, A A S u p, 95,9)$ report a CO survey on a 2 -arcminute grid over the Sgr A, B, and C source complexes. They revise their earlier model by concluding that the gas distribution in the inner few hundred parsecs is flat and untilted with respect to the Galactic plane, while the gas layer at larger radii flares and is warped, as in the earlier models. Zylka et al. (1992, AASup, 96, 525) surveyed the $6-\mathrm{cm} \mathrm{H}_{2} \mathrm{CO}$ absorption line out to a longitude of $4^{\circ}$, and along select strips, observed the $2-\mathrm{cm} \mathrm{H}_{2} \mathrm{CO}$ line as well. Particular attention is paid in their discussion to the "Clump 2 " cloud complex at $1=3.2^{\circ}$, which they decompose into "a number of kinematically well-defined sub-clouds." 


\title{
5.6 A Radio Transient Source
}

An intense radio transient source popped up in December 1990 within Sgr A West near the Galactic center (Zhao et al., 1992, Science, 255, 1538). Following its detection, it increased in intensity for about a month, and them declined for 3 months, all the while maintaining a steep radio spectrum. Zhao et al. suggest that the varying emission arose from a synchrotron-radiating plasma associated with an X-ray binary system.

\subsection{Sgr $A^{*}$}

Observational studies of the compact radio source at the Galactic nucleus, $\mathrm{Sgr} \mathrm{A*}$, and its near environment have led to some interesting suggestions, but solid breakthroughs in direct imaging will await the next triennium, when the full capacity of the VLBA is brought to bear on this source at wavelengths of $1 \mathrm{~cm}$ or less. The partially completed VLBA has already been used to reobserve Sgr A*: at $1.34 \mathrm{~cm}$, Lo et al. $(1993$, Nature, $\mathbf{3 6 2}, 38)$ confirm the previously inferred elliptical Gaussian model, with a major axis size of $2.4 \pm 0.2$ mas; at $7 \mathrm{~mm}$, Sgr $A^{*}$ is less than 0.4 mas (3.3 a.u.) in size (Backer et al., 1993, Science, in press; Krichbaum et al., 1993 AA, 274, L37). Since the radio size of $\mathrm{Sgr} \mathrm{A}^{*}$ is strongly affected by scattering off of electron density fluctuations along the line of sight, even higher frequencies will probably be required to probe the intrinsic source size. A lower limit to the size of $\mathrm{Sgr} \mathrm{A} \mathrm{A}^{*}$ is about $10^{12} \mathrm{~cm}$, derived from the observed limit to the refractive scintillation (Gwinn et al., 1991, ApJL, 381, L43). Sgr A* shows brightness variations, some of which may be attributable to intrinsic source variability (Zhao et al., 1991, in Relationships between AGN's and Starburst Galaxies, ed: A. Filippenko, in press).

One of the most interesting findings of the past triennium is that $\mathrm{Sgr} \mathrm{A}$ * has a strong excess of submillimeter emission (Zylka et al., 1992, AA, 261, 119; Dent et al., 1993, ApJ, 410, 650). The surviving explanation at present is that this is an ultracompact, self-absorbed synchrotron source. Serabyn et al. (1992, ApJL, 401, L87) observed this excess at $222 \mathrm{GHz}$ interferometrically, and confirmed the association of the submillimeter source with Sgr A*. A possible near-IR counterpart to Sgr A* was reported by Eckart et al. (1992, Nature, 355, 526), although a non-physical positional coincidence cannot yet be ruled out for this source.

The mass of $\mathrm{Sgr} \mathrm{A}^{*}$ (or of any putative black hole in its vicinity) is still unsettled. Ozernoy (1992, in Testing the AGN Paradigm, p 40, p 44) continues to produce arguments that any black hole in our Galactic nucleus must have a relatively low mass, perhaps $100-1000 \mathrm{M}_{\odot}$. This notion is supported by Sanders $(1992$, Nature, 359, 131), who points out that if the components of IRS16 are young stars which have formed recently within $0.1 \mathrm{pc}$ of the center, then there cannot be a very massive black hole there, because its tidal forces would have prevented star formation. On the other hand, Melia (1992, ApJL, 387, L25) finds that a million-M $\mathrm{M}_{\odot}$ model for Sgr A* involving a weak accretion flow out of the surrounding winds can reproduce the shape of the spectrum satisfactorily. In a related study, Melia et al. (1992, ApJL, 395, L87) describe how source size measurements at sub-cm wavelengths will be important for determining the mass of Sgr $A^{*}$. Current measurements already support a mass close to $10^{6} \mathrm{M}_{\odot}$. If $\operatorname{Sgr} \mathrm{A}^{*}$ is a massive black hole, then it should exert a noticeable gravitational lensing effect on some of the stars in the central stellar cluster (Wardle and Yusef-Zadeh 1992, ApJL, 387, L65).

\section{The High-Energy Component}

\author{
Hans Bloemen \\ SRON-Leiden / Leiden Observatory
}


This section summarizes studies of large-scale galactic phenomena in relation to gamma rays, $\mathrm{X}$-rays, cosmic rays, and magnetic fields.

\subsection{Gamma Rays}

The past triennium has seen a major step in gamma-ray astronomy with the launch of NASA's Compton Gamma-Ray Observatory in April 1991. The EGRET and COMPTEL telescopes have both performed an all-sky survey during the first 15 months of the mission. The large-scale studies that are of interest here have just started, but first results are available.

Preliminary findings from EGRET seem to confirm that the galactic radiation at energies above $\sim 50 \mathrm{MeV}$, previously studied with the SAS-2 and COS-B satellites, is largely of diffuse origin. However, many small-scale discrepancies between the EGRET observations and the modeled emission can be seen. These may be discrete gamma-ray sources, but a diffuse origin cannot be excluded. They make up less than $10 \%$ of the total emission from the galactic disk (Hunter et al. 1993, Proc. 23rd Int. Cosmic Ray Conf.). Of particular interest is the excess seen at the galactic centre, not coinciding with the peak of the CO emission (Mayer-Hasselwander et al. 1993, Proc. 23rd Int. Cosmic Ray Conf.). It is only seen at energies above $300 \mathrm{MeV}$, but it needs to be verified whether this is possibly due to the decreasing angular resolution of EGRET with decreasing energy.

The COMPTEL telescope provides for the first time good possibilities to map gamma-ray emission in the 1-30 MeV range, which also contains nuclear gamma-ray lines. The first $\mathrm{MeV}$ image of the inner Galaxy obtained with COMPTEL shows a clear ridge of emission along the Galactic plane, but it is not obvious that this emission is largely of diffuse origin (Bloemen et al. 1993, ApJS, ). The intensity distribution of this ridge distinctly differs from the expected distribution for a simple model of the diffuse radiation (i.e., a model similar to that used in the COS-B and EGRET studies).

COMPTEL has for the first time mapped the entire galactic plane in the light of the 1.809 $\mathrm{MeV}$ line from ${ }^{26} \mathrm{Al}$ radioactive decay (Diehl et al. 1993, Proc. Second Compton Symposium). The origin of ${ }^{26} \mathrm{Al}$ is still an unsolved problem. Various populations have been proposed as sources of ${ }^{26} \mathrm{Al}$, such as novae, supernovae of type II, and young massive stars (see e.g.review by Prantzos 1991, Gamma-Ray Line Astrophysics, p. 129). COMPTEL's imaging capability of the $1.809 \mathrm{MeV}$ emission will be very important to distinguish among the potential candidates.

COMPTEL has recently reached a real breakthrough in gamma-ray line spectroscopy with the discovery of intense 3-7 MeV emission from the Orion complex, which can be attributed to the cosmic-ray induced lines from ${ }^{12} \mathrm{C}^{\star}$ and ${ }^{16} \mathrm{O}^{\star}$ at 4.44 and $6.13 \mathrm{MeV}$, and with the discovery of the $1.15 \mathrm{MeV}^{44} \mathrm{Ti}$ line from the Cas-A supernova remnant.

The OSSE instrument aboard the Compton Observatory has performed numerous observations of the galactic plane and galactic-center region to measure the distribution of $511 \mathrm{keV}$ positron annihilation radiation and to search for time variability of the emission (Purcell et al.1993, ApJL, 413, L85). A strong line near $511 \mathrm{keV}$ and a positronium-like continuum are present. No time variability of the line flux has been observed. The OSSE data can be described by a point source within a few degrees of the galactic center (or a galactic bulge component), superimposed on a disk component producing significant emission at longitudes up to $\pm 20^{\circ}$.

Other observations of the $511 \mathrm{keV}$ line (and the low-energy continuum) were presented by Harris et al. $(1990, A p J, 363,135)$ [combination of SMM observations between 1980 and 1987], Gehrels et al. (1991, ApJL, 375, L13) [GRIS], and Chapuis et al. (1991, Gamma-Ray Line Astrophysics, p. 52) [HEXAGONE].

Skibo, Ramaty, and Leventhal $(1992, A p J, 397,135)$ and von Ballmoos (1991, ApJ, 380, 98) have presented further modeling of the distribution in the Galaxy of the $511 \mathrm{keV}$ emission and 
the implied positron annihilation.

\subsection{X-Rays}

The past triennium has seen the first fascinating results obtained with the ROSAT satellite. As part of the all sky survey, ROSAT has carried out the first complete scan of the galactic plane with unprecedented high sensitivity. First results for some sample areas presented by Motch et al. (1991, AA, 246, L24) illustrated the fantastic richness of these data. Full analysis of the observations will definitely provide important advances in our understanding of the (extended) galactic X-ray sources and the diffuse X-ray background.

Burrows and Mendenhall (1991, Nature, 351, 629) and Snowden et al. (1991, Science, 252, 1529) have reported on the ROSAT detection of shadowing of the X-ray background in the $1 / 4-$ $\mathrm{keV}$ band by the interstellar cloud in Draco, located a few hundred parsecs from the mid-plane. This suggests that the million-degree gas responsible for the X-ray emission may form a thick disk or halo around the Galaxy.

Snowden et al. $(1991, A p J, 364,11)$ have shown that the variations in the Wisconsin $\mathrm{B} / \mathrm{C}$ band intensity ratio is dipole-like and may be due to a temperature gradient in the X-ray emitting plasma of the local cavity. While the physical origin of such a temperature gradient is uncertain, the alignment of the dipole with the higher temperature (and absorbed) Loop I region may be significant.

Studies of the diffuse soft X-ray background (0.16-3.5 keV) as seen with the Einstein observatory were presented by Wu et al. (1991, ApJ, 379, 564) and Micela et al. (1991, ApJ, 380, 495). The intensity is found to be dependent on galactic longitude even when only high galactic latitude data are used. The Galactic ridge is not present at energies below $0.33 \mathrm{keV}$.

Garmire et al. (1992, ApJ, 399, 694) have presented the definitive maps of the diffuse X-ray background ( $0.5-2.8 \mathrm{keV})$ as surveyed by the low-energy detectors of the A-2 experiment aboard the HEAO 1 satellite.

McCammon and Sanders $(1990, A R A A, 28,657)$ have reviewed the soft X-ray background and its origins.

\subsection{Cosmic Rays and Magnetic Fields}

Galactic winds have played an important role in large-scale cosmic-ray propagation studies in this triennium. Breitschwerdt et al. $(1991, A A,, 245,79)$ discussed the mutual interaction of hot gas and cosmic rays escaping from the Galaxy. They found that beyond about $1 \mathrm{kpc}$ from the galactic plane, stationary outflow solutions do exist, provided that the intergalactic pressure is low. Pohl and Schlickeiser $(1990, A A,, \mathbf{2 3 9}, 424)$ have studied the propagation of cosmic rays under the influence of a galactic wind and investigated the potentials of radio-synchrotron observations to detect the presence of a galactic wind. Radio studies of cosmic rays in the Galaxy have been reviewed by Reich (1991, The Interstellar Disk-Halo Connection in Galaxies, p. 187). Webber, Lee, and Gupta $(1992, A p J, 390,96)$ and Bloemen et al. $(1993, A A, 267,372)$ compared direct observations of cosmic rays near Earth with the predictions from galactic wind models. They found that the data are consistent with a convection velocity $<20 \mathrm{~km} \mathrm{~s}^{-1}$ and that the effective cosmic-ray scale height in the solar vicinity has to be $\leqslant 3 \mathrm{kpc}$, independent of the presence of a galactic wind.

Ko, Dougherty, and McKenzie $(1991, A A, 241,62)$ studied the structure and stability of a static galactic halo including cosmic-ray and Alfvén-wave effects. Their findings lend support to the idea that such a halo cannot be stationary and that a steady outflow is indeed to be expected. On a global scale, however, a hydrostatic equilibrium seems plausible (Stephens 1991, The In- 
terstellar Disk-Halo Connection in Galaxies, p. 323). A galactic hydrostatic equilibrium with magnetic tension and cosmic-ray diffusion was studied by Boulares and Cox (1990, ApJ, 365, 544). They explored the structure of the $z$-distribution of mass and pressure in the solar neighborhood for three gravitational potentials, differing in their content of dark matter in the Galactic plane. An essential feature is that cosmic rays, magnetic fields, and a significant component of the interstellar mass all have high $z$-distributions characterized by scale heights $\geq 1 \mathrm{kpc}$.

Hartquist (1990, Evolution of the Interstellar Medium, p. 99) has reviewed the role of cosmic rays in interstellar dynamics. Ptuskin and Soutoul $(1990, A A, \mathbf{2 3 7}, 445)$ have discussed the propagation of cosmic rays in a cloudy interstellar medium. Particle acceleration and non-thermal radiation from $O B$ associations and superbubbles was studied by Bykov and Fleishman (1992, Sov. Astron. Lett. 8 and MNRAS, 255, 269). The origin of cosmic rays was reviewed by Axford (1991, Proc. 22nd Int. Cosmic Ray Conf., 6, 406).

\title{
7 The Chemical Evolution of the Galaxy
}

\author{
Francesca Matteucci \\ University of Trieste
}

\subsection{Light elements}

The study of light elements $\left(D,{ }^{3} \mathrm{He},{ }^{4} \mathrm{He},{ }^{7} \mathrm{Li},{ }^{9} \mathrm{Be}\right.$ and $\left.{ }^{11} \mathrm{~B}\right)$, which provides insights into both cosmological and Galactic history, has received a great deal of attention in the last three years. In particular, the measure of Be and B in metal poor stars of the Galactic Halo (Gilmore et al. 1991, $A p J, 378,17$, Ryan et al.1992, ApJ, 388, 184 and Gilmore et al.1992, Nature 357, 379) has posed serious problems concerning the production of these elements by cosmic-ray spallation on interstellar CNO nuclei. This finding encouraged speculation either that ${ }^{9} \mathrm{Be}$ could have been synthesized during an inhomogeneous big bang, or that some Galactic process other than cosmicray spallation might be involved (Pagel 1991, Nature 354, 267). However, the discovery of B in some of the same stars with B/Be ratio of about 10 (Duncan et al. 1992, ApJ, 401, 484) has revived the argument in favor of cosmic-ray spallation reactions in the interstellar medium. Prantzos et al. (1993, ApJ, 403,630) tried to explain Be and B in metal poor stars by suggesting that during the halo phase galactic cosmic rays were more confined than today and had flatter spectra at low energy. Very recently, Pagel (1993, in Workshop on "Cosmical Magnetism, ed. D. Lynden-Bell, Kluwer, in press) suggested a chemically inhomogeneous model for the halo. However, none of the proposed models is able to explain the observed linear relation between oxygen and beryllium abundances.

Although $\mathrm{Li}$ represents a key element in cosmology as it is one of the few nuclei which are synthesized during the big bang, it is still unclear what its primordial abundance was and how it varied during the Galactic lifetime. D'Antona and Matteucci (1991, $A A, 248,62)$ suggested novae and asymptotic-giant branch stars as possible stellar sites for ${ }^{7} \mathrm{Li}$ production. They also predicted an increase of a factor of 2 in the $\mathrm{Li}$ abundance during the last $4.5 \mathrm{Gyr}$. A recent paper (King 1993, $A J, 105,1087$ ) seemed to confirm this prediction. A study of possible ${ }^{7} \mathrm{Li}$ production sites was presented also by L.E. Brown $(1992, A p J, 389,251)$, who examined numerical chemical models for the evolution of $\mathrm{Li}, \mathrm{Be}, \mathrm{B}$ and $\mathrm{D}$. He concluded that the major producers of ${ }^{7} \mathrm{Li}$ are likely to be intermediate and high-mass stars $\left(M>4 M_{\odot}\right)$. He favored a primordial $\mathrm{Li}$ abundance of ${ }^{7} \mathrm{Li} / \mathrm{H}$ $\simeq 10^{-10}$. Reeves $(1993, A A, 269,166)$ discussed the $\mathrm{Li}$ isotopic ratio in the present Galactic ga (Lemoine et al.1992, AA, (MS1-642)) to derive information on the stellar source of ${ }^{7} \mathrm{Li}$. $\mathrm{He}$ ontained a yield for the last $4.5 \mathrm{Gyr}$ of $20 \mathrm{M}_{\odot}$ of ${ }^{7} \mathrm{Li}$ per Gyr per $10^{10} \mathrm{M}_{\odot}$ of gas. This amount can explain the difference between Population I and II abundances. Hobbs and Thornburn (1991, 
ApJ, 375, 116) measured $\mathrm{Li}$ abundance in six extreme metal poor dwarfs. Their results agreed with previous ones showing an invariance of the $\mathrm{Li}$ abundance in metal poor stars, so favoring a primordial origin for most or all the $\mathrm{Li}$ observed in these oldest stars. The observational status of $\mathrm{Li}$ has been reviewed very recently by Spite and Spite (1993, in Origin and Evolution of the Elements, p. 201) where the reader is directed also for the most recent results on abundances and nucleosynthesis.

Steigman and Tosi $(1992, A p J, 401,150)$ studied the Galactic evolution of $\mathrm{D}$ and ${ }^{3} \mathrm{He}$ in order to infer their primordial values, which provide a valuable test for big bang nucleosynthesis. They inferred upper and lower bounds to the primordial abundances of D and ${ }^{3} \mathrm{He}$ and used these bounds to derive constraints to the nucleon/photon ratio and to the number of new, light elementary particles.

\subsection{Heavy Elements}

High dispersion spectroscopy to measure the abundances of heavy elements in stars of the solar neighborhood has continued to be one of the most studied fields in these last three years. The major work in this field appeared recently (Edvardsson et al. 1993, $A A, \mathbf{2 7 5}, 101$ ) and it represents the result of 10 years work of coordinated observations and analyses by a team of six astronomers. They have studied the elemental composition of $189 \mathrm{~F}$ and $\mathrm{G}$ dwarfs in the solar neighborhood and derived the abundances of $\mathrm{O}, \mathrm{Na}, \mathrm{Mg}, \mathrm{Al}, \mathrm{Si}, \mathrm{Ca}, \mathrm{Ti}, \mathrm{Fe}, \mathrm{Ni}, \mathrm{Zr}, \mathrm{Ba}$, and $\mathrm{Nd}$. The relative iron abundance $[\mathrm{Fe} / \mathrm{H}]$ and the abundance ratios relative to iron of most elements are estimated to be accurate with a standard deviation of $0.05 \mathrm{dex}$. They have studied the evolution of $\alpha$-elements and odd-z elements, iron-peak elements and s-elements as a function of stellar age and orbital properties. They have confirmed that in general $\alpha$-elements are overabundant in metal poor stars, but in addition they have shown that $[\alpha / \mathrm{Fe}]$ for these metal poor stars decreases with increasing galactocentric distance, implying that the star formation was faster and started first in the central parts of the Galaxy. The abundances of $\mathrm{Na}$ and $\mathrm{Al}$ have been found to increase at different rates with metallicity in the disk, showing a connection to their respective production in $\mathrm{C}$ and $\mathrm{Ne}$ burning in massive stars. Finally they have found a big scatter in the age- $[\mathrm{Fe} / \mathrm{H}]$ relation and suggested that it could be due to inhomogeneous enrichment. On the other hand, Francois and Matteucci (1993, $A A$, in press) have suggested that part of this scatter can be due to the fact that some of the stars observed now in the solar region, might have been born at different galactocentric distances. The lack of a pronounced slope in the age- $[\mathrm{Fe} / \mathrm{H}]$ relation and the scatter was evident already from a previous work of Strobel (1991, $A A, \mathbf{2 4 7}, 35)$.

Abundances in extremely metal poor stars have been measured by Ryan et al.(1991, $A J$, 102, 303). They have shown that the trend for $\alpha$-elements, relative to $\mathrm{Fe}$, continues the trend observed in halo stars with $[\mathrm{Fe} / \mathrm{H}] \geq-2.5$. They have also shown that a large spread exists in the abundances of s-process elements in very metal poor stars. This has been confirmed by Molaro et al. (1993, Origin and Production of the Elements, p.476). Concerning the interpretation of the observed $[\alpha / \mathrm{Fe}]$ versus $[\mathrm{Fe} / \mathrm{H}]$ relations, usually interpreted as a time-delay effect due to the different lifetimes of stars giving rise to supernovae II and Ia, an alternative idea was proposed by Edmunds et al. (1991, MNRAS, 251,33p). In particular, they have proposed that the observed trend could be explained by a "pseudo-secondary" behavior of Fe. On the other hand, Matteucci and Francois (1992, $A A, \mathbf{2 6 2}, \mathrm{L1})$ continued proposing the time-delay effect and tried to fit the data from Bessel et al. (1991, ApJL, 383, L71) showing that the change in slope for the $[\mathrm{O} / \mathrm{Fe}]$ ratio occurs at $[\mathrm{Fe} / \mathrm{H}]=-1.7$ instead of at -1.0 . Their best model implies different time scales for the formation of the inner and the outer halo (see also Smecker-Hane and Wyse, 1992, $A J, \mathbf{1 0 3}, 621$, on the duration of the halo phase). This is again supporting the idea, also shared by Burkert et al. $(1992, A p J, 391,651)$, that the Galaxy formed from inside 
out. In particular, Burkert et al. presented a complex self-regulated chemo-dynamical model where heating and cooling processes in a multicomponent interstellar medium are taken into account. Ferrini et al. $(1992, A p J, 387,138)$ also presented a complex model for the evolution of the halo and disk in the solar neighborhood. Two phases for the gas are considered in the disk region (diffuse and molecular) and only one in the halo region (diffuse). Detailed chemical evolution is calculated confirming the time-delay interpretation for the $[\alpha / \mathrm{Fe}]$ versus $[\mathrm{Fe} / \mathrm{H}]$ trends. Josey and Tayler (1991, MNRAS, 251, 474) discussed the oxygen production in the solar neighborhood and derived constraints on the infall history.

Concerning the r- and s- process elements, Mathews et al. (1992, ApJ, 391, 719) studied the kinds of stellar environments responsible for $r$ - and $s$ - process nucleosynthesis by means of models of Galactic chemical evolution. The growth of $r$ - process appears to be associated to the ejection rate of material from massive stars. Low mass type II supernovae are favored if the r-process is primary. The growth of s-process material is consistent with production in intermediate mass stars. The yields of both $r$ - and s-process appear to require a neutron source independent of metallicity. Abundances of r-process elements in halo stars have been used as Galactic chronometers, in particular those of Th and Eu (see Francois, 1991 in The Stellar Populations of Galaxies, p.417, for the observed abundances and Pagel 1993, in Origin and Evolution of the Elements, p. 496, for the derivation of the age of the Galaxy). In particular, Pagel (1993) compared the data sets with different Galactic models and found that they agree with ages of the order of $15 \mathrm{Gyr}$. Sneden et al. $(1992, A A, 246,354)$ derived abundances of $\mathrm{Cu}$ and $\mathrm{Zn}$ in a sample of field and globular cluster stars. They found that $\mathrm{Zn}$ abundances closely follow the overall metallicities whereas there are $\mathrm{Cu}$ deficiencies in all metal poor stars. Matteucci et al. $(1993, A A, \mathbf{2 7 2}, 421)$ studied the evolutionary histories of $\mathrm{Cu}$ and $\mathrm{Zn}$ by means of a detailed Galactic chemical evolution model. They concluded that a satisfactory agreement with observations is reached only if one assumes that a substantial fraction of $\mathrm{Cu}$ and $\mathrm{Zn}$ has been produced by supernovae Ia.

\subsection{Abundance gradients in the Galactic disk}

Abundance gradients of single elements and ratios of elements represent useful constraints to stellar nucleosynthesis and models of Galactic chemical evolution. In Wilson and Matteucci (1992, $A R A A, 4,1)$ one can find an updated review on the interstellar abundances and Galactic abundance gradients. In this review new data are presented showing a gradient in ${ }^{12} \mathrm{C} /{ }^{13} \mathrm{C}$ and ${ }^{16} \mathrm{O} /{ }^{18} \mathrm{O}$ ratios with galactocentric distance. The presence of a gradient in the ${ }^{14} \mathrm{~N} /{ }^{15} \mathrm{~N}$ ratio is less clear and there is no measurable gradient of ${ }^{32} \mathrm{~S} /{ }^{34} \mathrm{~S}$ ratio. Theoretical predictions by Matteucci and D'Antona (1991, AA, 247, L37), where the contribution of novae to ${ }^{13} \mathrm{C}$ and ${ }^{15} \mathrm{~N}$ is taken into account, are compared to the data. The comparison seems to favor novae being the major progenitor responsible for the production of ${ }^{15} \mathrm{~N}$ and part of ${ }^{13} \mathrm{C}$. In the review it is also indicated that in the interstellar medium near the sun, the carbon isotope ratio is $\simeq 20 \%$ lower than the solar ratio. This shows that there has been only a moderate amount of enrichment of the nearby interstellar medium since the formation of the solar system. This result has been also confirmed by Centurion and Vladilo $(1992, A A, \mathbf{2 5 1}, 245)$. Friel and Janes $(1993, A A, 267,75)$ measured the iron gradient in the Galactic disk from old open clusters. Their result agrees with the gradient derived from younger open clusters, suggesting little change of the abundance gradient with time.

Finally, in Rana $(1991, A R A A, 29,129)$ one can find a review of the chemical evolution of the Galaxy. The author surveys the observational material concerning the G-dwarf metallicity distribution, the age-metallicity relation, gas and metallicity distributions in the Galaxy, the luminosity functions of stars and white dwarfs in the solar neighborhood, the distribution of pulsars etc.. 


\title{
7.4 Chemical Evolution of the Galactic Bulge
}

At the end of 1990 , Matteucci and Brocato ( $A p J, 365,539)$ predicted that the abundance ratios of $\alpha$-elements/Fe in bulge stars should be larger than solar over almost the full Fe range. This prediction was based on the time-delay effect and on the fact that in order to fit the distribution of bulge stars with metallicity (Rich, 1992, in The Center, Bulge and Disk of the Milky Way, p.47 and Geisler and Friel 1992, $A J, 104,128$ ) a fast time scale of star formation is required. In particular, the observed distribution of bulge stars shows that they form a distinct stellar population with age of the order of $10 \mathrm{Gyr}$ and abundance range from -1.5 to nearly $+1.0 \mathrm{dex}$. Very recently, Mc William and Rich (1993, preprint) presented the first detailed abundance study of galactic bulge $\mathrm{K}$ giants. From their analysis of the $\alpha$-elements relative to iron they concluded that $\mathrm{Mg} / \mathrm{Fe}$ and $\mathrm{Ti} / \mathrm{Fe}$ are enhanced by +0.3 dex relative to the solar value over almost the full $\mathrm{Fe}$ range, in very good agreement with the predictions. However, they also found that $\mathrm{Ca}$ and $\mathrm{Si}$ closely follow the normal trend of disk giants. Therefore, more observational and theoretical work seems to be required before assessing this point. Several evolutionary scenarios for the Bulge have been proposed but we are still not able to tell if the Bulge was the first Galactic structure to be formed, as argued by Lee(1991, IAU Symp. 149, p. 446) on the basis of the ages of the Bulge RR-Lyrae, or if the Bulge is younger than the globular clusters, as argued by Rich (1992) on the basis of the Bulge asymptotic giant branch stars. Wyse and Gilmore $(1992, A J, 104,144)$ also discussed possible evolutionary scenarios for the Bulge. In particular, they argued that its chemical distribution is best explained if most of the original mass were lost, and discussed the fate of this gas. They stressed the point that a way to distinguish between different mechanisms of Bulge formation is to look at abundance ratios.

\section{Stellar Dynamics}

\author{
James Binney \\ Oxford University
}

\subsection{The Dark Halo}

The radial extent of the dark halo continued to be a topic of interest. Kulessa \& Lynden-Bell (1992, MNRAS, 255, 105) inferred that it has density $\rho \sim r^{-2.4}$ and mass $M \approx 1.3 \times 10^{12}$ at $r<230$ kpc. Norris \& Hawkins 1991, ApJ, 380, 104 concluded from the radial velocities of horizontal-branch stars at galactocentric distances $r \approx 40-60 \mathrm{kpc}$ that the mass of the halo is probably proportional to $r$ out to at least $40 \mathrm{kpc}$, with $M(40 \mathrm{kpc}) \gtrsim 5 \times 10^{11} M_{\odot}$. Allen, Schuster \& Poveda (1993, AA, 244, 280) computed orbits for similar high-velocity stars. Kuhn (1993, ApJ, 409, L13) suggested that at least some of the dwarf spheroidal systems may not be gravitationally bound, but have unexpectedly long dispersal time on account of the nature of orbits in the dark halo. Some workers continued to explore the possibility that flat rotation curves arise not from extended matter distributions but from a failure of conventional dynamics at very small accelerations (Begeman, Broeils \& Sanders 1991, MNRAS, 249, 523).

Over the triennium a great deal of effort was devoted to the possibility of detecting the constituent objects of the dark halo by their ability to gravitationally lens background stars. Nemiroff $(1991, A A, 247,73)$ presented a comprehensive analysis of lensing statistics for dark halos composed of sub-stellar objects; these should be detectable provided the objects have masses in the range $10^{-8}-1 M_{\odot}$. If the rate of lensing events that follows from four recently reported events (Alcock et al., 1993, Nature, 365, 621, Aubourg et al., 1993, Nature, 365, 623) is correct, the halo must consist predominantly of sub-stellar objects. Richstone \& Gould (1992, ApJ, 388, 354) 
showed that sub-solar objects in the halo can be accompanied by extremely few objects massive enough to burn hydrogen; the overall halo mass-to-light ratio has to be $\Upsilon \gtrsim 2000 M_{\odot} / L_{\odot}$. Daly \& McLaughlin (1992, ApJ, 390,423) concluded that infrared observations with SIRTF or ISO should be able to detect a dark halo made of substellar objects providing these have mass $m \gtrsim 0.005 M_{\odot}$.

Hut \& Rees $(1993, M N R A S, 259,27 \mathrm{P})$ argued that the relatively low mass ( $m \lesssim 5 \times 10^{6} M_{\odot}$ ) of any black hole at the galactic centre might be incompatible with the proposal that the dark halo consists of massive $m>10^{5} M_{\odot}$ ) black holes; dynamical friction would drag such massive holes in to the centre at a significant rate, and, if they there merged into one another, the current limit of the mass of the central object would be exceeded. Moore (1993, ApJ, 413, L93) discusses the limits that can be placed on the masses of halo black holes by virtue of their capacity to disrupt globular clusters.

Boccaletti, Pucacco \& Ruffini (1991, AA, 244, 48), Hjorth \& Madsen (1991, MNRAS, 253, 703), Spergel \& Hernquist (1992, ApJ, 397, L75) and Kandrup, Mahon \& Smith (1993, AA, 271, $440)$ discussed various aspects of violent relaxation. The origin of the $r^{1 / 4}$-law now seems fairly well understood, and the incompleteness of violent relaxation is well documented. The significance of the short Liapunov exponents characteristic of N-body systems remains controversial, although the experiments of Sakagami \& Gouda $(1991, M N R A S, 249,241)$ argue that it is not significant macroscopically.

Evidence accumulated that large-scale fluctuations may be important in stellar systems. Miller \& Smith (1993, in Back to the Galaxy, eds. S.S. Holt \& F. Verter, AIP) reported surprisingly largeamplitude dipole distortions at the centres of stellar systems. Weinberg (1993, ApJ, 410, 543) showed that the large-scale modes of somewhat idealized models of stellar systems become highly excited as the systems' equilibria approach global instability, and that this excitation can greatly enhance relaxation.

Several workers extended the range of models of spherical stellar systems with analytic distribution functions (Gerhard 1991, MNRAS, 250, 812, Louis, Cuddeford 1991, MNRAS, 253, 414, Dejonghe \& Merritt 1992, ApJ, 391, 531, Louis 1993, MNRAS, 261, 283, Dehnen 1993, MNRAS, 256, in press). Saha (1991, MNRAS, 248, 492) refined and extended the standard approach to the stability of spherical systems and showed that systems previously thought to be immune to the bar instability are actually very slightly unstable. Papaloizou, Palmer \& Allen $(1992, M N$ $R A S, \mathbf{2 5 3}, 129)$ showed that slowly rotating spherical systems are unstable to the formation of a tumbling bar.

The dark halo is generally expected to be non-spherical but it is still unclear whether it is axisymmetric or triaxial. There was continued progress in the construction of models of hot, non-spherical stellar systems, although are knowledge of such systems remains primitive. Gerhard \& Saha (1991, MNRAS, 251, 449) and Binney \& Kumar (1993, MNRAS, 261, 584) explored techniques for obtaining approximate integrals for general gravitational potentials and Dehnen $\&$ Gerhard $(1993, M N R A S, 261,311)$ used one of these techniques to explore rather fully the possible internal dynamical structures of flattened, axisymmetric systems. Evans \& Lynden-Bell (1991, MNRAS, 251, 213) obtained similar models by solving the Jeans equations for systems with Stäckel potentials. Van der Marel (1991, MNRAS, 248, 515) used the Jeans equations and assumptions regarding the orientation of the principal axes of the velocity dispersion tensors of the metal-poor and dark halos to constrain the axis ratios of these two components.

Lees \& Schwarzschild (1992, ApJ, 384, 491) and Schwarzschild (1993 ApJ, 409, 563) showed that the halo could in principle be triaxial, although the dynamics of a triaxial halo would be fundamentally different from that of the classical Stäckel ellipsoids because the galactic potential is never quadratic in galactocentric distance. The phase space of a triaxial halo would be dominated by families of 'boxlet' orbits rather than the boxes and loops that fill the phase space of a Stäckel potential. It does not seem possible to construct strongly triaxial $(c / a \lesssim 0.6)$ halos from such 
boxlets. Dejonghe \& Laurent (1991, MNRAS, 252, 606) adapted Eddington's classical solution of the integral equation for the distribution function, $\rho(x)=\int d^{3} \mathbf{v} f(E)$, to the case of triaxial bodies embedded in a triaxial Stäckel potential. Batsleer \& Dejonghe $(1993, A A, 271,104)$ presented a self-consistent two-integral model whose density distribution and distribution function can both be written in terms of elementary functions.

\subsection{The Disk}

Kuijken \& Gilmore (1991, ApJ, 367, L9) refined their earlier estimate of the surface density $\Sigma_{0}$ of the disk at the Sun, concluding $\Sigma_{0}=48 \pm 9 \mathrm{M}_{\odot} \mathrm{pc}^{-2}$. It appears that most, and perhaps all, of the material in the disk is accounted for by known stars, remnants and interstellar gas (Kuijken 1991, ApJ, 372, 125, Bahcall, Flynn \& Gould, ApJ, 389, 234). Kuijken \& Gilmore (1991) find that the surface density of dark halo material within $1.1 \mathrm{kpc}$ of the plane is about $23 \mathrm{M}_{\odot} \mathrm{pc}^{-2}$.

The origin of the correlation between the velocity dispersions of stellar groups and their ages continued an active topic of debate. Burkert, Truran \& Hensler (1992, ApJ, 391, 651) revived the proposal that this correlation reflects the decay of turbulence and thus the scale-height of the interstellar gas layer. Sellwood \& Kahn $(1991, M N R A S$, 250, 278) discovered a self-propagating instability in $\mathrm{N}$-body disks, which cuts grooves in the disks by heating stars that are in resonance with the current pattern speed. It may be possible to find evidence for this kind of episodic heating in the phase-space distribution of disk stars (Sellwood 1993, in Galactic 8 Solar System Astrometry, ed. L. Morrison, CUP). Toth \& Ostriker (1992, ApJ, 389, 5) studied the heating of the disk by infalling satellites. In the Cold Dark Matter cosmology, large numbers of low-mass halos are predicted, and these may have small or negligible core radii. On falling into the Galaxy, such halos would do appreciable damage to the disk, and Toth \& Ostriker were able to place limits on their number density from the requirement that the disk remain pretty cool over several billion years.

The triennium saw major advances in the quality of the published observational data that can be compared with theoretical models of the evolution of the kinematics and chemical composition of the disk. (Fresneau, 1992, $A J$, 104, 578; Yoss, Bell \& Detweiler 1991, AJ, 102, 975, Evardson et al., 1993, $A A, \mathbf{2 7 5}, 101)$. Cameron \& Torra (1991, AA, 241, 57) determined Oort's constant $A$ from the motions of young stars and a derived a low value, $A=9.6 \mathrm{~km} \mathrm{~s}^{-1}$, after correcting for the effects of spiral structure. Jaschek \& Valbousquet $(1991, A A, \mathbf{2 4 2}, 77 ; 1992, A A, 255,124$; 1993, $A A, \mathbf{2 7 5}, 472$ ) determined the solar motion by a variety of techniques, finding it to be at $V=20 \pm 1 \mathrm{~km} \mathrm{~s}^{-1}$ in direction $\left.(l, b)=52 \pm 3^{\circ}, 22 \pm 3^{\circ}\right)$.

Cuddeford \& Binney $(1993, M N R A S, 265,000)$ investigated the discrepancy between the slopes of the circular speed curve, $v_{c}(R)$, at the Sun that are determined (a) directly and (b) from the ratio $\sigma_{\phi}^{2} / \sigma_{R}^{2}$ of the two velocity dispersions in the plane of the McCormick $\mathrm{K}$ and $\mathrm{M}$ stars. They showed that the classical relationship $\sigma_{\phi}^{2} / \sigma_{R}^{2}=-B /(A-B)$ is seriously in error, but that correcting this error actually increases the discrepancy between the directly and indirectly determined values of $d v_{c} / d R$. Kuijken \& Tremaine $(1993, A p J, \mathbf{x x x x}, \mathbf{x x x x})$ suggest that the discrepancy may arise because the disk is distorted into an ellipse by a triaxial halo potential. If the disk is elliptical, the Sun would have to lie close to a principal axis, and if that axis were the disk's major axis (the minor axis of the halo potential), the local value of $\sigma_{\phi}^{2} / \sigma_{R}^{2}$ would deviate from that predicted by the shape of the rotation curve in the observed sense. A more prosaic possibility is that the McCormick stars are for some reason unrepresentative: Meusinger, Reimann \& Stecklum $(1993, A A, \mathbf{2 4 5}, 57)$ find $\sigma_{\phi}^{2} / \sigma_{R}^{2}=0.55 \pm 0.2$ in better agreement with the value predicted from the circular-speed curve. Antonuccio-Delogu $(1991, A A, 247,45)$ fitted solutions of the Jeans equations to the radial run of velocity dispersion and found that the data were best fitted for the smallest allowed values of $\sigma_{\phi}^{2} / \sigma_{R}^{2}$. 
Robin, Crézé \& Mohan (1992, ApJ, 400, L25) show from deep CCD frames of the anticentre that the stellar disk stops abruptly $5.5-6 \mathrm{kpc}$ beyond the Sun.

\subsection{The Bulge}

As detailed elsewhere in this report, the triennium saw an upsurge of work on the bulge. Kent (1992, ApJ, 387, 181) presented an axisymmetric dynamical model of the bulge, and Minniti et al. (1992, ApJ, 393, L47) determined the variation with radius of the velocity dispersion $\sigma$ and rotation rate $V$ of the bulge from the motions of $\mathrm{K}$-giants. They find that $\sigma$ decreases with radius as predicted by Kent (1992), while $V$ is nearly proportional to $r$. Lindqvist, Habing \& Winnberg $(1992, A A, 259,118)$ determined the central angular velocity and mass profile of the bulge from the kinematics of OH/IR stars. Langevelde et al. (1992, AA, 261, L17) found OH/IR stars at the galactic centre with velocities as large as $-355 \mathrm{~km} \mathrm{~s}^{-1}$, and concluded that they are probably on elongated but bound orbits.

An important development was the confirmation by Combes et al. $(1990, A A, 233,82)$ and Raha, Sellwood and Kahn $(1991$, Nature, 352, 411) that the bar instability in a rapidly rotating disk is liable to lead to the disk thickening dramatically to form a mildly elongated and moderately flattened bar. The bulge may have been wholly or partly formed through the central part of the Galaxy's disk thickening in this manner. This picture would explain why many bulge stars are less than $10 \mathrm{Gyr}$ old, and why the bulge appears to be triaxial (Sellwood, 1993 in Back to the Galaxy, eds. S.S. Holt \& F. Verter, AIP).

Little and Carlberg (1991, MNRAS, 250, 161, MNRAS, 251, 227) and Hernquist \& Weinberg $(1992, A p J, 400,80)$ investigated the time-scale and effects of angular momentum transfer from a bar to the surrounding disk and halo. Analytical estimates indicate that a bar should feed energy and angular momentum into surrounding components, and this would be expected to lead to significant evolution of the bar. N-body experiments generally confirm the conclusions of analytic work, but suggest that the neglect in the latter of self-gravity causes the predicted angular momentum and energy transfers to be underestimated.

Two studies investigated the effect of triaxiality on the dynamical friction experienced by massive halo objects such as globular clusters. Pesce Capuzzo-Dolcetta \& Vietri (1992, MNRAS, 254, 466) studied the decay dynamical friction drives in the orbits of globular clusters belonging to a galaxy that resembles Schwarzschild's self-consistent triaxial system. Clusters on box orbits move through the dense central region of the galaxy and have decay times that can be as little as a tenth of the decay times of clusters on loop orbits of the same energy. Long Ostriker \& Aguillar $(1992, A p J, \mathbf{3 8 8}, 362)$ showed that a rapidly rotating bar, unlike Schwarzschild's non-rotating bar, would not drag globulars to the centre significantly more rapidly that a spherical bulge of similar radial density profile.

\subsection{Star clusters}

Capaccioli, Piotto \& Stiavelli $(1993, M N R A S, 261,819)$ find that the power-law indices, $x$, of globular cluster mass functions are better correlated with galactocentric distance than with metallicity, and interpret this finding as a consequence of the effect upon $x$ of disk shocking of the clusters. Hut (1993, ApJ, 403, 256) completed a survey of binary-single star interactions that covers the entire energy range of interest. Goodman \& Hut $(1993 A p J, 271,277)$ calculated the distribution of binaries that is in statistical equilibrium with a given field-star distribution. Lee \& Ostriker (1993, ApJ, 409, 617) found that the density at which binary-driven re-expansion of the core of a model globular cluster occurs depends sensitively numerical details, such as the Coulomb logarithm and time-step length employed. 
Theuns $(1992, A A, 259,503)$ modeled encounters between clusters of 500 stars and molecular clouds. He found that catastrophic encounters with the most massive stars limit cluster lifetimes to $<100 \mathrm{My}$.

\title{
9 Hydrodynamics
}

\author{
James Stone \\ University of Maryland
}

Hydrodynamic modeling has become an increasingly powerful tool for studies of the formation, structure, and dynamics of the Galaxy during the past triennium. Some of the major results from such studies are summarized below.

\subsection{Formation}

Sommer-Larsen (1991, MNRAS, 250, 356) described a self-similar analysis of the formation of the galactic disk by a protogalactic cooling inflow which reproduces a number of observations of the ages of stellar distributions. A simple chemical evolution model for a viscous galactic disk also reproduces the observed gas metallicity gradient (Hellsten \& Sommer-Larsen 1992, MNRAS, 255, 650 ). The self-regulated chemical and dynamical evolution of a hot protostellar disk has been examined by Berkert et al.(1992 ApJ, 391, 651), who found that the observed vertical structure of the disk was reproduced in a natural way as a consequence of gravitational settling.

\subsection{Halo}

The formation, vertical structure, and hydrodynamic stability of the galactic halo continued to be an important problem during the triennium. Ko et al. (1991, $A A, \mathbf{2 4 1}, 62)$ and Ko $(1991, A A, 242$, $85)$ studied the structure and stability of a static galactic halo including contributions to the total pressure from the thermal plasma, cosmic rays, and Alfvén waves. At large distances from the galactic plane, the model predicts a pressure much larger than that of the ambient intergalactic medium, implying the halo is not static but drives a steady wind. In the solar neighborhood, Boulares \& Cox $(1990, A p J, 365,544)$ concluded magnetic fields, cosmic rays and a significant component of the interstellar mass all have high $z$-distributions, characterized by scale heights $\geq$ $1 \mathrm{kpc}$. By studying the propagation of cosmic ray nuclei in a convecting halo, Webber et al. (1992, $A p J, 390,96)$ set a limits on the thickness of the halo at less than $4 \mathrm{kpc}$ and a galactic wind velocity of less than $20 \mathrm{~km} \mathrm{~s}^{-1}$ within this distance. Einaudi \& Ferrara (1991, ApJ, 371, 571) have given steady state solutions of the MHD equations applicable to galactic wind models, emphasizing the importance of conditions in the disk for determining the properties of the flow. Fichtner et al. $(1991, A p J, 371,98)$ have considered the effects of nonspherical flow geometries on galactic wind models. Shapiro (1991, The Interstellar Disk-Halo Connection in Galaxies, p.417) has summarized the results of new studies of the galactic fountain model, in which supernova heated gas in the galactic disk escapes into the halo, radiatively cools, and condenses into dense clouds which fall back to the disk. Shapiro \& Benjamin $(1991$, PASP, 103, 923) have reported that optically thin, steady fountain flows in which the effects of nonequilibrium ionization/recombination and photoionization are included can reproduce the observed properties of UV absorption and emission lines. Raymond $(1992, A p J, \mathbf{3 8 4}, 502)$ has found reconnection in the random component of the galactic magnetic field in the halo may be a significant additional source of heating if models of impulsive energy release in solar microflares can be scaled to conditions in the halo. Danly (1992, $P A S P, 104,819)$ has reported significant variations in the infalling halo gas in the northern 
galactic hemisphere, indicating multidimensional effects may be important (Houck \& Bregman $1990, A p J, 352,506)$. In analyzing the evidence for the inflow of neutral gas toward the disk, Mirabel (1990, Structure and dynamics of the interstellar medium, p.396) concludes most infalling gas in the solar vicinity originated in the disk as part of the disk-halo circulation with only a moderate component originating from extragalactic sources. Wakker (1991, The interstellar disk-halo connection in galaxies, p.27) reviewed the observed properties of High Velocity Clouds (HVCs). Ferrara \& Einaudi $(1992, A p J, 385,475)$ used a linear stability analysis of the MHD equations to study the formation of HVCs in various background equilibrium models for the halo. Comerón \& Torra $(1992, A A, 261,94)$ used two dimensional time-dependent simulations to study the oblique impact of an HVC on the galactic disk, suggesting the Gould Belt may have resulted from such a process. Meyerdierks $(1991, A A, 251,269)$ provided extensive comparisons between observations of the North Celestial Pole Loop with a theoretical description of a cloudGalaxy collision. Li \& Ikeuchi $(1992, A p J, 390,405)$ have performed two dimensional simulations of galactic chimneys, calculating the distribution and ballistic motions of HVCs formed in the radiatively cooled gas.

\subsection{Superbubbles}

Following the remarkable observations of galactic "worms" and superbubbles catalogued by Koo et al. (1992 ApJ, 390, 108), a number of authors have undertaken studies of the hydrodynamical growth and blowout of supershells from the disk. Igumentshchev et al. $(1990, A A, \mathbf{2 3 4}, 396)$ reported two dimensional hydrodynamical simulations of the blowout of supershells. Fragmentation of the shell by Rayleigh-Taylor instabilities in the blowout phase is evident. Tomisaka has reported two dimensional $(1992, P A S J, 44,177)$ and fully three dimensional $(1990, A p J L, \mathbf{3 6 1}$, L5) magnetohydrodynamic simulations of the blowout of superbubbles including the effect of an ordered magnetic field of $5 \mu \mathrm{G}$ in the plane of the disk. Such fields are able to impede the growth of superbubbles across field lines, confining them to the disk $(z<200 \mathrm{pc})$ without restricting growth along the fields lines. These results have interesting implications for the filling factor of hot gas from superbubbles in the disk.

\subsection{Dynamos and the Magnetic Field}

An outstanding problem in galactic gas dynamics continues to be the operation of the dynamo, as evidenced by the large number of papers on this subject published during the triennium. The global properties of the galactic magnetic field were reviewed by Trimble (1990, Galactic and Intergalactic Magnetic Fields, p.29), while reviews of dynamo theory were given by Ruzmaikin (1990 ibid) and Krause \& Wielebinski (1991, Rev. in Modern Astro., 4, 260). Vallée (1991, ApJ, $366,450)$ has matched observations of field reversals in the spiral arms of the Galaxy to azimuthal, radial, and vertical modes of turbulent dynamo theory, allowing a prediction of the mean field direction in the 'Perseus + I' arm at $12 \mathrm{kpc}$. Ferrière $(1992, A p J, 389,286 ; 1992, A p J, 391$, 188) has investigated the action of supernova and superbubbles on generating radial field from azimuthal field (the $\alpha$-effect). A detailed study of the $\alpha \omega$-dynamo by Parker (1992, ApJ, 401, 137 ) indicates azimuthal fields are generated primarily in the gaseous disk, while the $\alpha$-effect and field dissipation via rapid reconnection occurs in magnetic lobes in the halo inflated by cosmic ray pressure. By considering dynamo amplification of initially weak fields, Kulsrud \& Anderson (1992, $A p J, 396,606)$ concluded it is difficult to generate the galactic mean field from an initially very weak seed field. new linear and nonlinear dynamo models for a galactic disk embedded in a halo were obtained by Brandenburg et al. (1992, AA, 259, 453). A number of authors considered the stability of the magnetized galactic disk. Hanawa et al. (1992, PASJ, 44, 509) investigated both 
Parker and Jeans instabilities in the disk by means of a linear analysis. Matsumoto \& Shibata (1992, PASJ, 44, 167) used time-dependent MHD simulations to study the nonlinear growth of the Parker instability in three dimensions. Gomez de Castro \& Pudritz (1992, ApJ, 395, 501) used a number of observational data sets to argue that the motion of the Taurus molecular cloud and other structures in the Gould Belt has been initiated by an odd mode of the Parker instability.

\title{
9.5 Galactic Center
}

Stark et al. (1991, MNRAS, 248, 14P) have argued that molecular clouds near the Galactic Center must be losing angular momentum rapidly either via dynamical friction or interaction with a barred potential, and must therefore be spiraling inward. Yasutami \& Tatematsu $(1990, P A S J, 42,517)$ reached a similar conclusion for clouds on a much larger scale. Gerhard (1991, Dynamics of Disc Galaxies, p.111) and Binney et al. (1991, MNRAS, 252, 210) argue that a rapidly rotating bar in fact dominates the dynamics of cold gas near the Galactic Center. This conclusion is supported by comparison of the hydrodynamics of $\mathrm{CO}$ gas in the Galaxy with other galaxies known to be barred spirals (Nakai 1992, PASJ, 44, L27). Chevalier (1992, ApJL, 397, L39) has modeled the hydrodynamics of the wind from the galactic Center, and finds the associated shocked wind bubble can produce magnetic structures which may be related to those observed. The strong wind from Sgr A* appears to have ablated a remarkable "tail" of ionized gas from the cool supergiant IRS 7, as reported by high resolution VLA radio observations (Yusaf-Zadeh \& Morris 1991, ApJL, 371, L59; Yusaf-Zadeh \& Melia 1992, ApJL, 385, L41) and optical images (Serabyn et al.1991, $A p J, 378,557)$. From the observed properties of molecular gas at the Galactic Center, Spergel \& Blitz $(1992$, Nature, 357, 665) conclude the ambient gas pressure is some two-and-a-half orders of magnitude higher than in the solar neighborhood. Wardle \& Königl (1990, ApJ, 362, 120) have modeled the generation of a wind at the Galactic Center by an accretion disk threaded by open axial magnetic field lines. Observed magnetic field strengths within $70 \mathrm{pc}$ of the center are large enough to significantly affect the dynamics of the molecular clouds (Morris 1990, Galactic and intergalactic magnetic fields, p.361) and spiral density waves (Tagger 1991, it Dynamics of disc galaxies, p. 117). In a review of supersonic jets from many systems in the Galaxy, Padman et al. (1991, Beams and jets in astrophysics, p.484) have given a "tourist's guide" to possible hydrodynamic jets in the Galactic Center region.

\section{Dynamics and Kinematics from Eastern Europe}

\author{
Alexei Fridman \\ Institute of Astronomy RAS, Moscow
}

\subsection{Kinematics and Linear Dynamics (General Questions)}

A steady state galaxy model consisting of a halo and a disk was considered by S. Kutuzov (Abstracts of the conference: Mathematical Methods in Structuring of the Structure and Dynamics of Gravitating Systems, Petrozavodsk, June 15-18, 1993). The halo does not rotate and has an isotropic velocity distribution, the disk rotates and has an anisotropic asymmetrical velocity distribution. A phase density of the disk is assumed to be linear function of the angular momentum (area integral) with coefficients depending on the energy integral.

A linear analysis of the stability of a thin non-self-gravitating gaseous disk shows (A. Morozov, V. Mustsevoi, and A. Prosvirov, 1992, Sov. Astron. Let., 18, 20) that for a continuous radial dependence of the rotation rate, a second unstable mode may be excited in addition to the previously known centrifugal instability mode. It is suggested that one possible reason for the formation of 
branching arms in spiral galaxies may be the simultaneous excitation and superposition of the two modes.

E. Malkov, T. Nuzhnova, and B. Sagintaev (1991, Sov. Astron. Let., 17, 200) investigated the stability of gravitating elliptical disks embedded in a spherical subsystem with respect to largescale bending and bar-like perturbations. It is shown that a sufficiently massive external subsystem can stabilize an elliptical disk of practically any eccentricity with internal flows corotating with the disk.

A theorem is proved on the number of instabilities of a uniform gravitating medium consisting of $n$ components in relative motion (A. Fridman and Yu. Fridman, 1991, Sov. Astron. Let., 17, 74).

A two-parameter family of parabolic orbits in the meridional galactic plane was found by E. Timoshkova (1991, Sov. Astron., 35, 660) in the problem of perturbed stellar motion in a rotationally-symmetric field of a galaxy. The relationship between the family and stationary solutions is established. Analytic expressions for star co-ordinates and velocities are obtained in closed form for periodic and asymptotic motion along the trajectories.

The estimates of time of stare transitions between halo and core in spherical clusters as result of stellar encounters were given by V. Danilov (Kinematics Phys. Celest. Bodies, 1991, 7, 75). Numerical investigation of grass-evolution equations for quasi-stationary and nonstationary (in regular field) stellar clusters has been performed. There are three possible types of "halo-core" structure evolution in the quasi-stationary stellar clusters depending upon the initial conditions. The results of comparison of the author's cluster model calculations with observations and numerical $\mathrm{N}$-body experiments are discussed.

\subsection{Nonlinear Dynamics (General Questions)}

Solitary vortices in a stellar subsystem of a galactic disk are obtained on the framework of collisionless stellar hydrodynamics approximation (V. Korchagin and A. Ryabtsev, 1991, AA, 246, $368)$.

V. Dolotin and A. Fridman (1991, Sov. Phys. JETP, 72, 1) derived a nonlinear equation describing the dynamics of perturbations of small but finite amplitude of a solid-body rotating gaseous system of nonuniform density. In the case of short-wave perturbations (of wavelength $\lambda$ much smaller than the Jeans wavelength $\lambda_{J}$, i.e. $\lambda \ll \lambda_{J}$ ) and in the opposite limiting case of long-wavelength perturbations $\lambda \gg \lambda_{J}$ the equation is reduced to the well-known nonlinear equation for Rossby waves with a vector nonlinearity - Charney-Obukhov (or Hasegawa-Mita) equation. The weak turbulence spectrum of this equation leads to known observable relation between the fundamental parameters of gas population of the Galaxy. The stationary solution of this equation describes dipolar vortices (modons). Possible observable manifestations of modons (double galaxies, double galactic nuclei and double stars) are discussed (1991)

V. Afanasiev, S. Dodonov, A. Sumin, A. Fridman (1991, Asrton. Tsirk, N1551, 3) established by the numerical simulations of the dynamics of gaseous disk with a rotation curve corresponding the observed one for galaxy Mrk 1040, the generation of two anticyclonic vortices, the morphology and localization of which are close to that discovered recently by 6-meter telescope in SAO.

Effects of nonstationary action on the dynamical evolution of non-interacting particles, which are in a certain regular field, were studied by A. Baranov (1991, Astron. Zh., 68, 1160). Various forms of the disturbance of the spiral-wave type with slowly changing parameters have been considered. Qualitative features of the action of the non-stationary disturbance have been revealed. There is a category of captured stars which move together with the resonant zone and fill the phase space in the domains, not contiguous to each other. The noncaptured stars pass in the intervals between these domains and are not subject essentially to the action of the disturbance. 


\subsection{Structure, Kinematics, and Dynamics of the Milky Way}

Seventeen giant (170-700 pc) star-gas complexes (SGCs) have been detected by T. Sitnik ( $D y$ namics of Galaxies and their Molecular Cloud Distributions, 1991, p.91) within $3 \mathrm{kpc}$ from the Sun. These SGCs include stellar groupings younger than (2-3) $\times 10^{7}$ years and molecular clouds with masses $10^{5}-10^{6} M_{\odot}$, embedded into HI superclouds. An investigation of seven large-scale SGCs has shown, within them, an age gradient of stellar groups, equal to $(0.3-1.2) \times 10^{7}$ years for distances 270-500 pc. The sequential changing of the ages of stellar groupings across the Sgr-Car arm (with the youngest stellar groups, all the young $\mathrm{H}_{2} \mathrm{O}$ masers and $\mathrm{CO}$ clouds located at the inner boundary of the arm) is evidence for the formation of this arm by a spiral density wave. This wave, propagating in individual HI superclouds with molecular clouds inside, stimulates star formation therein. Crude estimates of the spiral pattern angular velocity yield $\Omega_{p} \approx 17-25 \mathrm{~km}$ $\mathrm{s}^{-1} \mathrm{kpc}^{-1}$ and the corotation radius $\approx 8.8-13 \mathrm{kpc}$. Perhaps the Cygnus arm is lying near the corotation radius, since there is no age gradient across this arm. The direction of the changing ages is different in all the Cas-Per arm's SGCs. It cannot be excluded that in one of these SGCs a "reverse" age gradient is observed.

A new method of determination of the radio halo size is suggested by A.Lazaryan and G. Chibisov (1991, Sov. Astron. Let., 17, 208) based on a statistical study of synchrotron fluctuations where HII regions are used as distance indicators.

A. Sumin, A. Fridman, and V. Haud (1991, Pis'ma Astron. Zh., 17, 779) proposed on the basis of observational data the seven-component model of the Galaxy with sharp jump of the gas component's surface density (two orders of magnitude) near the outer edge of molecular ring. Within this model the partial contributions of each subsystem to the model rotational curve of the Galaxy were calculated. It turns out that the value of dimensionless growth rate of hydrodynamical instability for the gas component only, being much less than unity, is very close to that for the same gas in the gravitational field of the whole Galaxy. From this fact two conclusions follow: 1) The unstable gas component of the Galaxy lies near the boundary of the hydrodynamical instability in accordance with the Le Chatelier principle. 2) The stellar population of the Galaxy probably does not effect dynamically the generation of the spiral structure in the gaseous component. A. Sumin, A. Fridman, and V. Haud (ibid. 698) also proposed the three component model of the Galaxy and revealed that this model is dynamically close to the seven-component model, and therefore it makes possible to use this more simple model to study the different problems of the Galaxy dynamics.

The distribution of the peculiar velocity components in the galactic coordinate for 207 of the main sequence stars was considered by B. Fesenco (Abstracts of the conference: Mathematical Methods in Structuring of the Structure and Dynamics of Gravitating Systems, Petrozavodsk, June $15-18,1993$ ) with dividing of that sequence into 13 subintervals. The observed distribution is in good agreement with the normal low distribution. On the base of the Moscow high precision photometric catalogue the sample of 116 solar type stars is formed. The normal law distribution for the peculiar velocity components is confirmed. The velocity of the sun relative to the stars of that sample is equal to $34 \pm 5 \mathrm{~km} \mathrm{~s}^{-1}$. The value of the standard deviation of the peculiar velocity components averages $26 \mathrm{~km} \mathrm{~s}^{-1}$. The distribution of the stars in fields of equal area is not in agreement with Poisson law. 